\title{
Radio continuum emission in the northern Galactic plane: Sources and spectral indices from the THOR survey ${ }^{\star}$
}

\author{
Y. Wang ${ }^{1}$, S. Bihr ${ }^{1}$, M. Rugel ${ }^{1}$, H. Beuther ${ }^{1}$, K. G. Johnston ${ }^{2}$, J. Ott ${ }^{3}$, J. D. Soler ${ }^{1}$, A. Brunthaler ${ }^{4}$, \\ L. D. Anderson ${ }^{5,6,7}$, J. S. Urquhart ${ }^{8}$, R. S. Klessen ${ }^{9,10}$, H. Linz ${ }^{1}$, N. M. McClure-Griffiths ${ }^{11}$, S. C. O. Glover ${ }^{9}$, \\ K. M. Menten ${ }^{4}$, F. Bigiel ${ }^{9}$, M. Hoare ${ }^{2}$, and S. N. Longmore ${ }^{12}$ \\ 1 Max-Planck Institute for Astronomy, Königstuhl 17, 69117 Heidelberg, Germany \\ e-mail: wang@mpia.de \\ 2 School of Physics and Astronomy, University of Leeds, Leeds LS2 9JT, UK \\ 3 National Radio Astronomy Observatory, PO Box O, 1003 Lopezville Road, Socorro, NM 87801, USA \\ ${ }^{4}$ Max-Planck-Institut für Radioastronomie, Auf dem Hügel 69, 53121 Bonn, Germany \\ 5 Department of Physics and Astronomy, West Virginia University, Morgantown, WV 26506, USA \\ 6 Adjunct Astronomer at the Green Bank Observatory, PO Box 2, Green Bank, WV 24944, USA \\ 7 Center for Gravitational Waves and Cosmology, West Virginia University, Chestnut Ridge Research Building, Morgantown, \\ WV 26505, USA \\ ${ }^{8}$ School of Physical Sciences, University of Kent, Ingram Building, Canterbury, Kent CT2 7NH, UK \\ 9 Universität Heidelberg, Zentrum für Astronomie, Institut für Theoretische Astrophysik, Albert-Ueberle-Str. 2, 69120 Heidelberg, \\ Germany \\ 10 Universität Heidelberg, Interdisziplinäres Zentrum fur Wissenschaftliches Rechnen, INF 205, 69120 Heidelberg, Germany \\ 11 Research School of Astronomy and Astrophysics, The Australian National University, Canberra, ACT, Australia \\ 12 Astrophysics Research Institute, Liverpool John Moores University, IC2, Liverpool Science Park, 146 Brownlow Hill, Liverpool \\ L3 5RF, UK
}

Received 14 June 2018 / Accepted 16 August 2018

\begin{abstract}
Context. Radio continuum surveys of the Galactic plane can find and characterize $\mathrm{H}$ II regions, supernova remnants (SNRs), planetary nebulae (PNe), and extragalactic sources. A number of surveys at high angular resolution $\left(\leq 25^{\prime \prime}\right)$ at different wavelengths exist to study the interstellar medium (ISM), but no comparable high-resolution and high-sensitivity survey exists at long radio wavelengths around $21 \mathrm{~cm}$.

Aims. Our goal is to investigate the $21 \mathrm{~cm}$ radio continuum emission in the northern Galactic plane at $<25^{\prime \prime}$ resolution.

Methods. We observed a large percentage of the Galactic plane in the first quadrant of the Milky Way $\left(l=14.0-67.4^{\circ}\right.$ and $\left.|b| \leq 1.25^{\circ}\right)$ with the Karl G. Jansky Very Large Array (VLA) in the C-configuration covering six continuum spectral windows (SPW). These data provide a detailed view on the compact as well as extended radio emission of our Galaxy and thousands of extragalactic background sources.

Results. We used the BLOBCAT software and extracted 10916 sources. After removing spurious source detections caused by the side lobes of the synthesized beam, we classified 10387 sources as reliable detections. We smoothed the images to a common resolution of $25^{\prime \prime}$ and extracted the peak flux density of each source in each SPW to determine the spectral indices $\alpha\left(\operatorname{assuming} I(v) \propto v^{\alpha}\right)$. By cross-matching with catalogs of $\mathrm{H}_{\text {II }}$ regions, SNRs, PNe, and pulsars, we found radio counterparts for $840 \mathrm{H}_{\text {II }}$ regions, 52 SNRs, 164 $\mathrm{PNe}$, and 38 pulsars. We found 79 continuum sources that are associated with X-ray sources. We identified 699 ultra-steep spectral sources $(\alpha<-1.3)$ that could be high-redshift galaxies. About 9000 of the sources we extracted are not classified specifically, but based on their spatial and spectral distribution, a large percentage of these are likely to be extragalactic background sources. More than 7750 sources do not have counterparts in the SIMBAD database and more than 3760 sources do not have counterparts in the NED database.

Conclusions. Studying the long wavelengths centimeter continuum emission and the associated spectral indices allows us to characaterize a large percentage of Galactic and extragalactic radio sources in the area of the northern inner Milky Way. This database will be extremely useful for future studies of a diverse set of astrophysical objects.
\end{abstract}

Key words. catalogs - surveys - radio continuum: general - techniques: interferometric

\footnotetext{
* The full continuum catalog, the full Table 4 , and all the fits files of the continuum data are available at the project website http://www2 . mpia-hd.mpg.de/thor/DATA/www/, and at the CDS via anonymous ftp to cdsarc.u-strasbg.fr (130.79.128.5) or via http:// cdsarc.u-strasbg.fr/viz-bin/qcat?J/A+A/619/A124
}

\section{Introduction}

A number of surveys at high angular resolution $\left(\leq 20^{\prime \prime}\right)$ at multiple wavelengths exist to study the interstellar medium (ISM), from infrared (e.g., UKIDSS ${ }^{1}$, Lucas et al. 2008;

\footnotetext{
1 UKIRT Infrared Deep Sky Survey.
} 
Spitzer/GLIMPSE ${ }^{2}$, Benjamin et al. 2003; Churchwell et al. 2009, Spitzer/MIPSGAL ${ }^{3}$, Carey et al. 2009) to submillimeter/millimeter (e.g., ATLASGAL ${ }^{4}$ and BGPS $^{5}$, Schuller et al. 2009; Rosolowsky et al. 2010; Aguirre et al. 2011; Csengeri et al. 2014) and radio (e.g. MAGPIS ${ }^{6}$, COR$\mathrm{NISH}^{7}$, Helfand et al. 2006; Hoare et al. 2012) wavelengths. Previously, the best $21 \mathrm{~cm} \mathrm{H}$ I line survey was the HI Very Large Array Galactic Plane Survey (VGPS, Stil et al. 2006), which has a resolution of $60^{\prime \prime}$, significantly more coarse than the resolution of the aforementioned surveys. This was one of the motivations for initiating The $\mathrm{H} \mathrm{I}, \mathrm{OH}$, Recombination line survey of the Milky Way (THOR ${ }^{8}$; Beuther et al. 2016). Using the Karl G. Jansky Very Large Array (VLA) in C-configuration, we achieve a spatial resolution of $<25^{\prime \prime}$. The WIDAR correlator at the VLA allows us to observe many spectral lines simultaneously, in particular several molecular $\mathrm{OH}$ transitions, a series of $\mathrm{H} n \alpha$ radio recombination lines (RRLs; $n=151$ to 186 ), and eight spectral windows (SPWs) to cover the continuum emission between 1 and $2 \mathrm{GHz}$ (Bihr et al. 2015, 2016; Beuther et al. 2016; Rugel et al. 2018). We observed a large percentage of the Galactic plane in the first quadrant of the Milky Way $\left(l=14.0-67.4^{\circ}\right.$ and $\left.|b| \leq 1.25^{\circ}\right)$ in several semesters (from 2012 to 2014). The continuum data from the first half of the survey $\left(l=14.0-37.9^{\circ}\right.$ and $\left.l=47.1-51.2^{\circ}\right)$ have been published by Bihr et al. (2016). In this paper, we combine all the continuum data and present the results from the full survey.

The radio continuum emission from 1 to $2 \mathrm{GHz}$ is dominated by thermal free-free emission from electrons and non-thermal synchrotron emission of the relativistic electrons in magnetic fields (e.g., Wilson et al. 2013). These can be distinguished by the spectral index $\alpha$, assuming $I(v) \propto v^{\alpha}$, where $I(v)$ is the intensity at frequency $v$. The thermal free-free emission shows an almost flat $(\alpha=-0.1)$ spectrum if it is optically thin or a positive spectral index if it is optically thick with values varying between -0.1 and 2 (e.g., Keto 2003; Wilson et al. 2013). H II regions and planetary nebulae $(\mathrm{PNe})$ are often the sources for thermal freefree emission. In contrast to this, synchrotron emission shows a negative spectral index whose value depends, amongst other things, on the particle energy distribution. Synchrotron emission with a spectral index $\alpha<-0.5$ is often found toward extragalactic jets powered by an active galactic nucleus (AGN; e.g., Hey 1971; Rybicki \& Lightman 1979). Galactic supernova remnants (SNRs) often show synchrotron emission with a spectral index around -0.5 (e.g., Bhatnagar et al. 2011; Green 2014; Reynoso \& Walsh 2015). Thus the spectral index can help us to characterize the nature of the continuum sources we detected in the survey. This allows us to determine whether they are Galactic or extragalactic, which is crucial for $\mathrm{H}$ I and $\mathrm{OH}$ absorption studies.

Compact Galactic radio sources associated with X-ray emission are usually pulsar wind nebulae (e.g., Brisken et al 2005; Miller et al. 2005) or X-ray binaries (XRB or microquasars; Mirabel \& Rodríguez 1998, 1999). By investigating the $\mathrm{X}$-ray and radio flux ratios, the spectral indices and observations at other (optical/infrared) wavelengths of the Galactic sources in detail, we can also constrain the type of the

\footnotetext{
Galactic Legacy Infrared Midplane Survey Extraordinaire.

A 24 and 70 Micron Survey of the Inner Galactic Disk with MIPS.

APEX Telescope Large Area Survey of the Galaxy.

Bolocam Galactic Plane Survey.

6 Multi-Array Galactic Plane Imaging Survey.

the Co-Ordinated Radio "N" Infrared Survey for High-mass star formation.

8 http://www.mpia.de/thor/Overview.html
}

sources, i.e., low mass XRB, PN, and pulsars (e.g., Seaquist 1993; Maccarone et al. 2012; Tetarenko et al. 2016). With the high angular resolution $\left(<25^{\prime \prime}\right)$ of our THOR continuum data, we not only can derive the spectral indices of the sources, but also further study the variation in frequency and space.

The paper is structured as follows: In Sect. 2, we present the observations and data reduction. Sect. 3 presents the methods we used to extract sources and determine the spectral indices. Section 4 describes the continuum catalog and the distribution of the continuum sources we extracted. The nature of continuum sources are discussed in Sect. 5. The conclusions and summary are presented in Sect. 6 . The appendix gives additional information of the continuum observations and tables.

\section{Observations and data reductions}

We observed a part of the first quadrant of the Galactic plane $\left(l=14.0-67.4^{\circ}\right.$ and $\left.|b| \leq 1.25^{\circ}\right)$ with the Karl G. Jansky Very Large Array (VLA) in C-configuration at $L$ band from 1 to $2 \mathrm{GHz}$. The detailed observing strategy and data reduction is discussed and described in Bihr et al. (2016) and Beuther et al. (2016). With the WIDAR correlator, we covered the H I $21 \mathrm{~cm}$ line, $4 \mathrm{OH}$ lines, $19 \mathrm{H} \alpha$ recombination lines, and eight continuum bands, i.e., SPWs. Each continuum SPW has a band width of $128 \mathrm{MHz}$. Owing to strong contamination from radio frequency interference (RFI), two SPWs around 1.2 and $1.6 \mathrm{GHz}$ were not usable and discarded. The remaining six SPWs are centered at $1.06,1.31,1.44,1.69,1.82$, and $1.95 \mathrm{GHz}$. For the fields at $l=23.1-24.3^{\circ}$ and $25.6-26.8^{\circ}$, the SPW around $1.95 \mathrm{GHz}$ is also severely affected by RFI and is therefore flagged (see Bihr et al. 2016).

The calibration and imaging were performed with the CASA $^{9}$ software package. We employed the RFlag algorithm, which was first introduced to AIPS by E. Greisen in 2011 to flag outliers in each visibility dataset before imaging. For imaging, we chose a pixel size of $2.5^{\prime \prime}$ and robust $=0$ as a weighting parameter, which results in a synthesized beam size varying from $9^{\prime \prime}$ to $25^{\prime \prime}$ depending on the SPW and the declination of various observing blocks and $u v$-coverage (see Table 1 for details). We also used the multiscale CLEAN in CASA to recover the large scale structure better, and chose the scale parameters as 1,3 , and 6 times the resolution element. The cleaning process was set to stop at a threshold of $5 \mathrm{mJy}^{\text {beam }}{ }^{-1}$ or $10^{5}$ iterations, whichever was reached first. The noise of our data is dominated by the artifacts resulting from residual side lobes, and varies from $\sim 0.3$ to $>1 \mathrm{mJy}^{-1}$ beam ${ }^{-1}$ depending on the frequency and sky position. The thermal noise is $\sim 0.1 \mathrm{mJy} \mathrm{beam}^{-1}$. We discuss the noise level of the images in Sect. 3.

THOR+VGPS data: The $1.4 \mathrm{GHz}$ continuum data from the VGPS survey (Stil et al. 2006) combined VLA D-configuration with single-dish observations from Effelsberg and have an angular resolution of $60^{\prime \prime}$. The $14.0^{\circ}<l<17.5^{\circ}$ Galactic longitude range in the VGPS data is comprised of single-dish observations. We smoothed the THOR continuum data at $1.4 \mathrm{GHz}$ to a resolution of 25 " and used the task "feather" in CASA to combine the THOR data with those from VGPS. While this combined dataset retains the high angular resolution of the THOR observations, it can recover the large scale structure. The combined image of the whole survey at $1.4 \mathrm{GHz}$ in Fig. 1 shows that large scale $\mathrm{H}$ II regions and SNRs dominate the extended radio emission in the inner Galactic plane $\left(l<55^{\circ}\right)$, while compact sources are distributed across the whole survey area (more prominent in

\footnotetext{
9 http://casa.nrao.edu
} 
Table 1. Synthesized beams of SPWs.

\begin{tabular}{lcc}
\hline \hline SPW & $\begin{array}{c}\text { Frequency range } \\
(\mathrm{MHz})\end{array}$ & Restoring beam size \\
\hline spw-1060 & $989-1117$ & $24.4^{\prime \prime} \times 15.1^{\prime \prime}$ to $15.1^{\prime \prime} \times 14.7^{\prime \prime}$ \\
spw-1310 & $1244-1372$ & $19.7^{\prime \prime} \times 12.5^{\prime \prime}$ to $12.6^{\prime \prime} \times 12.2^{\prime \prime}$ \\
spw-1440 & $1372-1500$ & $18.1^{\prime \prime} \times 11.1^{\prime \prime}$ to $12.0^{\prime \prime} \times 11.6^{\prime \prime}$ \\
spw-1690 & $1628-1756$ & $15.4^{\prime \prime} \times 9.1^{\prime \prime}$ to $9.8^{\prime \prime} \times 9.5^{\prime \prime}$ \\
spw-1820 & $1756-1884$ & $14.5^{\prime \prime} \times 8.9^{\prime \prime}$ to $9.2^{\prime \prime} \times 9.1^{\prime \prime}$ \\
spw-1950 & $1884-2012$ & $13.1^{\prime \prime} \times 8.1^{\prime \prime}$ to $8.6^{\prime \prime} \times 8.2^{\prime \prime}$ \\
averaged image & spw-1440 \& spw-1820 & $18.1^{\prime \prime} \times 11.1^{\prime \prime}$ to $12.0^{\prime \prime} \times 11.6^{\prime \prime}$ \\
THOR+VGPS & 1420 & $25^{\prime \prime} \times 25^{\prime \prime}$ \\
\hline
\end{tabular}

Notes. Averaged images are used for source extraction. The size of the restoring beam varies owing to declination and $u v$-coverage differences among observing blocks.

area $l>55^{\circ}$ ). These compact sources are most likely extragalactic sources, and we discuss them later. Anderson et al. (2017) used this dataset and identified 76 new Galactic SNR candidates in the survey area. Although the spectral band of the VGPS continuum $(\sim 1 \mathrm{MHz})$ is different from our THOR continuum data $(\sim 128 \mathrm{MHz})$, Anderson et al. (2017) showed that the flux retrieved from the combined data is consistent with the literature by comparing the flux density of the known SNRs (Green 2014). All the reduced continuum data, including the THOR+VGPS dataset, can be accessed from the THOR survey website located at http://www . mpia.de/thor and are available at the CDS.

\section{Source extraction and spectral index determination}

While the combined THOR+VGPS dataset exists for the $1.4 \mathrm{GHz}$, for the other bands only the THIR data are available. Therefore, all spectral index analysis is performed on the THORonly data. To achieve the best signal-to-noise ratio $(\mathrm{S} / \mathrm{N})$, we chose the two SPWs, spw-1820 and spw-1440, that are least affected by RFI and have the lowest noise, smoothed them into the same resolution, and then averaged them for source extraction. Since the noise of our data is dominated by side-lobe noise, we followed the method described in Hales et al. (2012) and Bihr et al. (2016) and constructed noise maps using the averaged residual image from the clean process. The noise maps are shown in Fig. A.1. We furthermore calculated the cumulative percentage noise level map area at a specific $7 \sigma$ noise level in mJy beam ${ }^{-1}$ (Fig. 2). While the lowest $7 \sigma$ noise level $\left(\leq 1 \mathrm{mJy}\right.$ beam $^{-1}$, dominated by thermal noise) is achieved in $\sim 20 \%$ of the survey area, more than $60 \%$ of the survey area has a $7 \sigma$ noise level $\leq 2 \mathrm{mJy}$ beam $^{-1}$. Compared to the first half of the survey (Bihr et al. 2016), the noise is better for the entire survey because in the large longitude Milky Way regions beyond Galactic longitudes of roughly $51^{\circ}$ there are fewer strong sources (Fig. 1 and Fig. A).

We used the software BLOBCAT (Hales et al. 2012) to extract the sources from the averaged continuum images. Following the same criteria as in Bihr et al. (2016) for the first half of the survey, we set the detection threshold as $5 \sigma$ and the flooding threshold to the standard value of $2.6 \sigma$ (Hales et al. 2012), and extracted 10916 sources. We then inspected each source visually, identified 530 sources as obvious observational side-lobe artifacts, and removed them from the catalog. The remaining 10387 sources should be mostly real detections. It was difficult to determine whether some sources with a $\mathrm{S} / \mathrm{N}$ of 5 to $7 \sigma$ were real.
Therefore, we considered sources with a $\mathrm{S} / \mathrm{N}$ higher than $7 \sigma$ with higher confidence. In total, out of the 10916 extracted sources, 7521 sources were detected with a $\mathrm{S} / \mathrm{N}$ higher than $7 \sigma, 2866$ have a $\mathrm{S} / \mathrm{N}$ of 5 to $7 \sigma, 530$ are observational side-lobe artifacts.

Following the same method described in Bihr et al. (2016), we performed a completeness test by extracting artificial compact sources from a $0.5^{\circ} \times 0.5^{\circ}$ region with a constant noise level. The result shows that we detected $94 \%$ of all sources with a peak intensity above $7 \sigma$ (Fig. 3). Combining the noise map, we constructed completeness maps for different peak intensities; these maps are shown in Appendix B.

Since our observations cover a wide range in frequency from $1 \mathrm{GHz}$ to $2 \mathrm{GHz}$, we can determine the spectral indices for the sources we identified, assuming $I(v) \propto v^{\alpha}$. To do this, we first smooth all SPWs to a common resolution of $25^{\prime \prime}$, then extract the peak intensities. Since the integrated flux density suffers more from filtering effects for extended sources, we use only the peak intensity for the spectral index determination. We use the SciPy function "curve_fit" to fit the peak intensity between 1 to $2 \mathrm{GHz}$ of each source we extracted and derive the spectral index $\alpha$ defined as $I(v) \propto v^{\alpha}$ together with the uncertainty of $\alpha$. Since a single SPW image has a lower $\mathrm{S} / \mathrm{N}$ than the averaged image we used for source extraction, we choose a lower threshold $(3 \sigma)$ for spectral index determination. We fit only the peak intensities that are higher than three times of the noise level of the respective SPW, i.e., a reliable intensity (see also Bihr et al. 2016). For 8228 sources we can extract a reliable peak intensity from at least two SPWs and derive a spectral index. We list the number of SPWs that were used for spectral index determination in the continuum source catalog as "fit_spws". For instance, fit_spws $=6$ means the peak intensity for all six SPWs is reliable and used for spectral index determination, fit_spws $=4$ means the peak intensity for only four SPWs is reliable and used for spectral index determination. Figure 4 shows the distribution of the uncertainties of the determined spectral index for all sources and for sources with a different number of SPWs. This clearly demonstrates that the more SPWs used to fit the spectral index, the smaller the uncertainty is. The mean uncertainty of the spectral indices are $\Delta \alpha=0.18,0.43,0.70,1.1,2.2$ for sources detected in $6,5,4,3$, and 2 SPWs, respectively. We consider all spectral indices fitted with four or more SPWs (fit_spws $\geq 4$ ) reliable.

\section{Catalog}

Our continuum catalog contains 31 columns for each source as described in detail in Table 2. We summarize the number of 

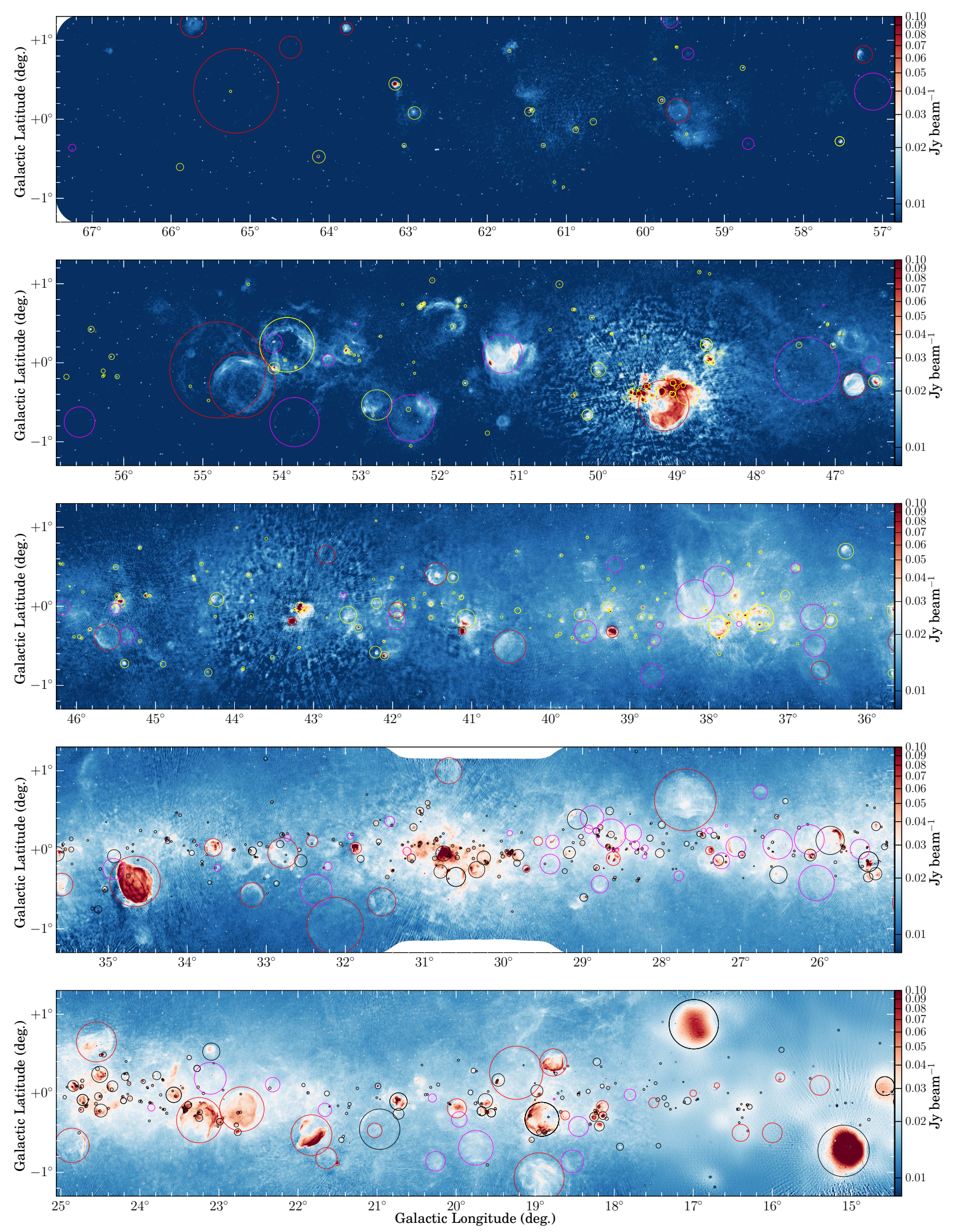

Fig. 1. THOR+VGPS $1.4 \mathrm{GHz}$ continuum map of the whole survey. The yellow and black circles denote the WISE H in regions (Anderson et al. 2017) matched with our continuum sources (see also Sect. 5.3). The red circles indicate the SNRs from Green (2014). The magenta circles indicate the new SNR candidates identified in this combined dataset (Anderson et al. 2017). The synthesized beam size is $25^{\prime \prime}$. For regions $l<17.5^{\circ}$, complementary D-configuration data do not exist. Therefore, in these regions we have only combined the THOR data with the Effelsberg data. 
Y. Wang et al.: Radio continuum emission in the northern Galactic plane: Sources and spectral indices from the THOR survey

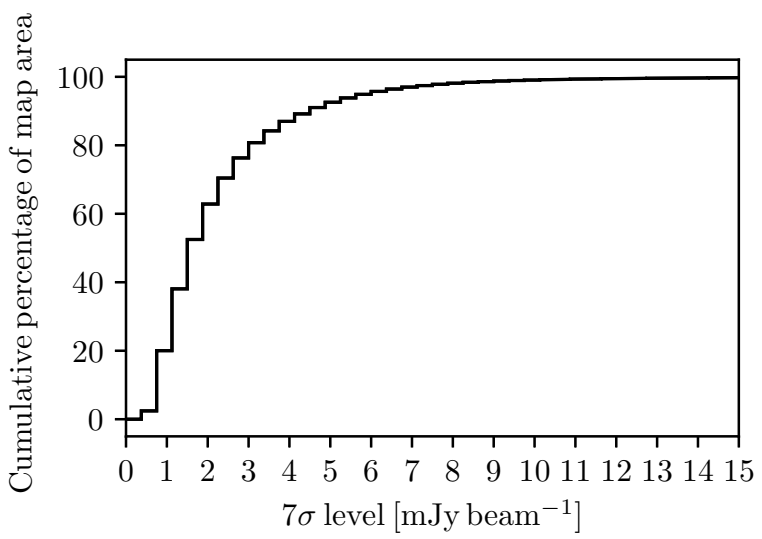

Fig. 2. Cumulative percentage noise level diagram. The percentage of the map area as a function of the noise level at a $\mathrm{S} / \mathrm{N}$ of $7 \sigma$ in $\mathrm{mJy}_{\text {beam }}{ }^{-1}$. More than $60 \%$ of the survey area has a noise level of $7 \sigma \lesssim 2 \mathrm{mJy}$ beam $^{-1}$.

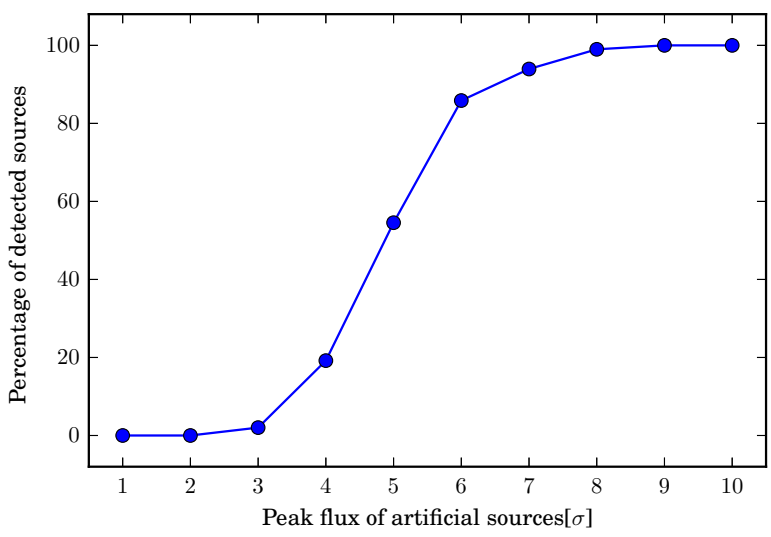

Fig. 3. Completeness test plot. Percentage of the added artificial sources detected as a function of the source peak intensity in units of the noise level $\sigma$.

extracted sources in Table 3. The BLOBCAT algorithm identifies compact sources close to each other or superimposed on large extended emission as one single source. Additionally, large extended sources, such as SNRs, can be separated into several different sources. As the two examples shown in Fig. 5, two H II regions are identified into one single object in our catalog and the SNR W44 is resolved into 18 separate objects. Therefore, the exact numbers in Table 3 should be treated cautiously. We crossmatched the THOR continuum catalog with catalogs of different types of Galactic sources (H II regions, SNRs, etc.; see Sect. 5) and list all the matched counterparts. For a source matched with multiple sources, we also list the number of counterparts that the continuum source is associated with in column "Ncounter" in the catalog (Table 2). We also categorise sources in groups based on counterparts they share, i.e., sources associated with the same H II regions or SNRs are in the same group with a "GroupID". The size of the groups are also listed as "GroupSize" (Table 2). In total, 126 sources have more than 1 counterpart and 153 sources are categorised into 49 groups with GroupSize varying from 2 to 19. We discuss the association between the different Galactic and extragalactic sources in detail in Sect. 5.

The distribution of the extracted sources along Galactic longitude and latitude is shown in Fig. 6. The majority (79\%; Table 3) of the extracted sources are unresolved, and a weak gradient for

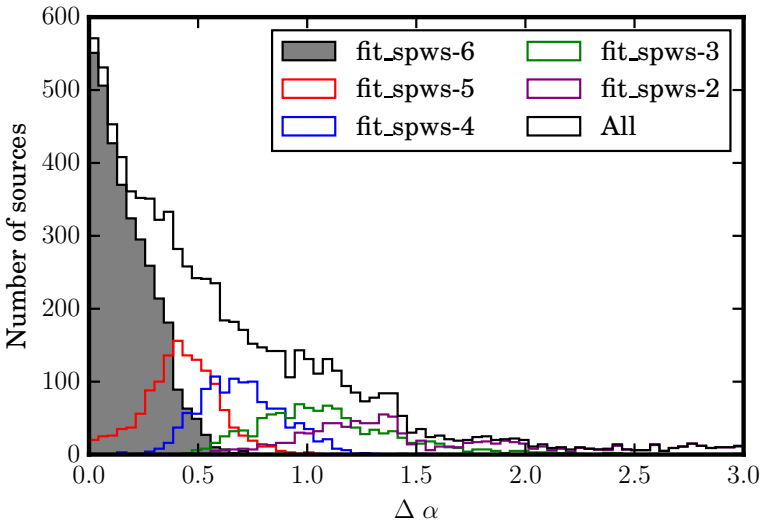

Fig. 4. Histogram of the uncertainties of the determined spectral indices. The black line includes all sources for which we are able to determine a spectral index; the gray shaded area represents the sources for which we have an intensity measurement in all 6 SPWs, and therefore the spectral index $\alpha$ is derived from fitting all 6 SPWs; the red line includes sources fitted with 5 SPWs; the blue line includes sources fitted with 4 SPWs; the green line includes sources fitted with 3 SPWs; and the purple line includes sources fitted with 2 SPWs.

the those sources along Galactic longitude is shown in Fig. 6 , where more weak unresolved sources are identified in the larger longitude regions. The resolved sources are evenly distributed along Galactic longitude, but larger sources $\left(\mathrm{n}_{\mathrm{pix}}>500\right.$; effective radius of $\sim 32^{\prime \prime}$ ) are more concentrated in the inner Galaxy, since these are mostly Galactic $\mathrm{H}_{\text {II }}$ regions and SNRs. Another possible reason is that there are more mergers of smaller sources in the inner longitudes because of higher source density and longer path lengths through the Galaxy. The distribution along Galactic latitude reveals that the resolved sources are concentrated close to the Galactic midplane, whereas the distribution of the unresolved sources shows a dip at $|b|<0.5^{\circ}$. The distribution of both resolved and unresolved sources drops at $|b|>1.0^{\circ}$ owing to higher noise at the edges of the survey area.

We did a Kolmogorov-Smirnov test (KS test) for the dip in the distribution of the unresolved sources at $|b|<0.5^{\circ}$. We first re-binned the source counts into 100 bins and measured the mean value and standard deviation of the distribution at $|b|>0.5^{\circ}$ and $|b|<1.0^{\circ}$. Then we generated a random artificial distribution with the same mean value and standard deviation. Finally we computed the KS statistic and $p$-value between the random artificial and real distribution with the SciPy function "ks_2samp". The mean KS statistic and $p$-value for 100 run is $0.34 \pm 0.041$ and $0.001 \pm 0.0018$, respectively. Thus the dip at $|b|<0.5^{\circ}$ is statistically significant. A similar distribution along Galactic latitude is also found in the first half of our survey (Bihr et al. 2016) and in the MAGPIS survey (Helfand et al. 2006). Fewer unresolved sources are identified at $|b|<0.5^{\circ}$ and in the inner Galaxy, which could be because the strong emissions from the extended Galactic sources such as H II regions and SNRs lower our detection completeness to weak sources toward the Galactic midplane and inner Galaxy (see Appendix B).

\section{Discussion}

\subsection{Comparison with other surveys}

The comparison between the peak positions of the common sources in the THOR (first half, $l=14.0-37.9^{\circ}$ and $l=47.1-$ 51.2 ${ }^{\circ}$ ), MAGPIS (Helfand et al. 2006), and CORNISH surveys 
Table 2. Description of the continuum source catalog entries.

\begin{tabular}{|c|c|c|c|}
\hline Col. Num. & Name & Unit & Description \\
\hline 1 & Gal. ID & & Name of the source the form G"Gal. longitude" \pm "Gal. latitude" $a$. \\
\hline 2 & RA & deg & Right ascension in $\mathrm{J} 2000$ of the peak position. \\
\hline 3 & Dec & deg & Declination in J2000 of the peak position. \\
\hline 4 & S_p $p^{b}$ & $\mathrm{Jy} \mathrm{beam}^{-1}$ & Peak intensity of the aver. image used for source extraction (see Sect. 3). \\
\hline 5 & S_int & Jy & Integrated flux density of the averaged image (see Sect. 3). \\
\hline 6 & $\mathrm{~S} / \mathrm{N}$ & & Signal-to-noise ratio in the averaged image. \\
\hline 7 & BMAJ & $\operatorname{arcsec}$ & Major axis of the synthesized beam used for source extraction. \\
\hline 8 & BMIN & $\operatorname{arcsec}$ & Minor axis of the synthesized beam used for source extraction. \\
\hline 9 & $\mathrm{BPA}$ & $\operatorname{deg}$ & Position angle of the synthesized beam used for source extraction. \\
\hline 10 & n_pix & & Number of pixels flooded by BLOBCAT (see Sect. 3). \\
\hline 11 & resolved_source & & Resolved source label (see Sect. 3). 1 = Resolved, $0=$ Point. \\
\hline 12 & S_p $(\text { spw-1060) })^{c}$ & $\mathrm{Jy} \mathrm{beam}^{-1}$ & Peak intensity around $1.06 \mathrm{GHz}$ used for spectral index (see Sect. 3). \\
\hline 13 & delta_S_p(spw-1060) ${ }^{c}$ & $\mathrm{Jy} \mathrm{beam}^{-1}$ & Uncertainty of peak intensity around $1.06 \mathrm{GHz}$. \\
\hline 14 & S_p $(\text { spw-1310 })^{c}$ & $\mathrm{Jy} \mathrm{beam}^{-1}$ & Peak intensity around $1.31 \mathrm{GHz}$ used for spectral index (see Sect. 3). \\
\hline 15 & delta_S_p(spw-1310) ${ }^{c}$ & $\mathrm{Jy} \mathrm{beam}^{-1}$ & Uncertainty of peak intensity around $1.31 \mathrm{GHz}$. \\
\hline 16 & S_p $\left(\right.$ spw-1440) ${ }^{c}$ & Jy beam ${ }^{-1}$ & Peak intensity around $1.44 \mathrm{GHz}$ used for spectral index (see Sect. 3). \\
\hline 17 & delta_S_p(spw-1440) ${ }^{c}$ & $\mathrm{Jy} \mathrm{beam}^{-1}$ & Uncertainty of peak intensity around $1.44 \mathrm{GHz}$. \\
\hline 18 & S_p $(\text { spw-1690) })^{c}$ & $\mathrm{Jy} \mathrm{beam}^{-1}$ & Peak intensity around $1.69 \mathrm{GHz}$ used for spectral index (see Sect. 3). \\
\hline 19 & delta_S_p(spw-1690) ${ }^{c}$ & $\mathrm{Jy} \mathrm{beam}^{-1}$ & Uncertainty of peak intensity around $1.69 \mathrm{GHz}$. \\
\hline 20 & S_p (spw-1820) ${ }^{c}$ & $\mathrm{Jy} \mathrm{beam}^{-1}$ & Peak intensity around $1.82 \mathrm{GHz}$ used for spectral index (see Sect. 3). \\
\hline 21 & delta_S_p(spw-1820) ${ }^{c}$ & $\mathrm{Jy} \mathrm{beam}^{-1}$ & Uncertainty of peak intensity around $1.82 \mathrm{GHz}$. \\
\hline 22 & S_p $\left(\right.$ spw-1950) ${ }^{c}$ & $\mathrm{Jy} \mathrm{beam}^{-1}$ & Peak intensity around $1.95 \mathrm{GHz}$ used for spectral index (see Sect. 3). \\
\hline 23 & delta_S_p(spw-1950) ${ }^{c}$ & $\mathrm{Jy} \mathrm{beam}^{-1}$ & Uncertainty of peak intensity around $1.95 \mathrm{GHz}$. \\
\hline 24 & alpha & & Spectral index of source we derived (see Sect. 3). \\
\hline 25 & delta_alpha & & Uncertainty of spectral index. \\
\hline 26 & fit_spws & & Number of SPWs used to fit the spectral index (see Sect. 3). \\
\hline 27 & Note $^{d}$ & & "HII", "SNR_green", "SNR_anderson", "PN", "PSR", "Xray", “jets"(see Sect. 5). \\
\hline 28 & Counterparts & & The counterparts of the $\mathrm{H}$ II region ${ }^{e}, \mathrm{SNR}^{f}$, the planetary nebula ${ }^{g}$ and the pulsars ${ }^{h}$. \\
\hline 29 & Ncounter & & The total number of counterparts that the continuum source is associated with. \\
\hline 30 & GroupID & & The Group of continuum sources associated with one or more same counterparts. \\
\hline 31 & GroupSize & & The number of the continuum sources in the same group. \\
\hline
\end{tabular}

Notes. ${ }^{(a)}$ Indicating the peak position. ${ }^{(b)}$ The synthesized beam is different for different fields and is given in rows 7-9. ${ }^{(c)}$ The synthesized beam is smoothed to $25^{\prime \prime} \times 25^{\prime \prime} .{ }^{(d)}$ We classified the continuum source into different categories. "HII": sources associated with H II regions from Anderson et al. (2014); "SNR_green": sources associated with SNRs from Green (2014); "SNR_anderson": sources associated with SNR candidates from Anderson et al. (2017); "PN": sources classified as planetary nebula; "PSR": sources classified as pulsars; "Xray": sources associated with X-ray sources; “jets”: sources classified as extragalactic jets candidates. ${ }^{(e)}$ Anderson et al. (2014). ${ }^{(f)}$ Green (2014), Anderson et al. (2017). ${ }^{\left({ }^{g}\right)}$ Parker et al. (2016). ${ }^{(h)}$ Manchester et al. (2005).

Table 3. Numbers of the catalog.

\begin{tabular}{lcc}
\hline \hline Description & Numbers & Percentage \\
\hline All & 10387 & $100 \%$ \\
$\mathrm{~S} / \mathrm{N}>7 \sigma$ & 7521 & $72 \%$ \\
Resolved sources & 2210 & $21 \%$ \\
Unresolved sources & 8177 & $79 \%$ \\
$n_{\text {pix }}>500$ & 439 & $4 \%$ \\
fit_spws $\geq 4$ & 5857 & $56 \%$ \\
\hline
\end{tabular}

(Hoare et al. 2012; Purcell et al. 2013) presented in Bihr et al. (2016) shows that the THOR survey has positional accuracy better than 2.5" (full width at half maximum of the Gaussian fit to the position offset distribution; see Fig. 15 in Bihr et al. 2016). Considering that the pixel size of our continuum images is only $2.5^{\prime \prime}$, the spatial accuracy of the THOR survey is consistent with previous observations.

To check for consistency in the flux density, we compared the THOR with MAGPIS. Within our survey area $\left(14.2^{\circ}<l<67.4^{\circ}\right.$, $b<|1.25|^{\circ}$ ), the MAGPIS catalog contains 2256 discrete sources.
We cross-matched our catalog with the MAGPIS catalog with a matching radius of $5^{\prime \prime}$, and we found 1440 matches. We then selected sources with the following criteria: 1) $S / N>3$ in spw$1440,2)$ unresolved in the THOR catalog, and 3) major axis smaller than $10^{\prime \prime}$ in the MAGPIS catalog. We selected 735 sources using the aforementioned criteria. We compared the $1.4 \mathrm{GHz}$ continuum flux density from the spw-1440 in THOR and the flux densities in MAGPIS, as shown in Fig. 7. The MAGPIS/THOR flux ratio shows a tight distribution around 1 with a median value of $\sim 0.97$. Most of the sources are within $5 \sigma$ from the flux ratio equals to one. Sixty-seven sources show a deviation larger than $7 \sigma$ from one, among which 56 sources have a ratio smaller than one. If we compare the integrated flux from MAGPIS to the peak flux from THOR for these 56 sources, the mean ratio is 1.08. This indicates that these sources could have been resolved by MAGPIS. For the remaining 11 sources the ratios are smaller than 1.18 , therefore we cannot rule out the possibility of variability for these sources. Among these 11 sources, 2 are matched with X-ray sources (ratios are 1.02 and 1.08; see also Sect. 5.7). The remaining 9 sources do not match any identified source and they all have negative spectral indices, which suggests that they can be extragalactic sources. 
Y. Wang et al.: Radio continuum emission in the northern Galactic plane: Sources and spectral indices from the THOR survey
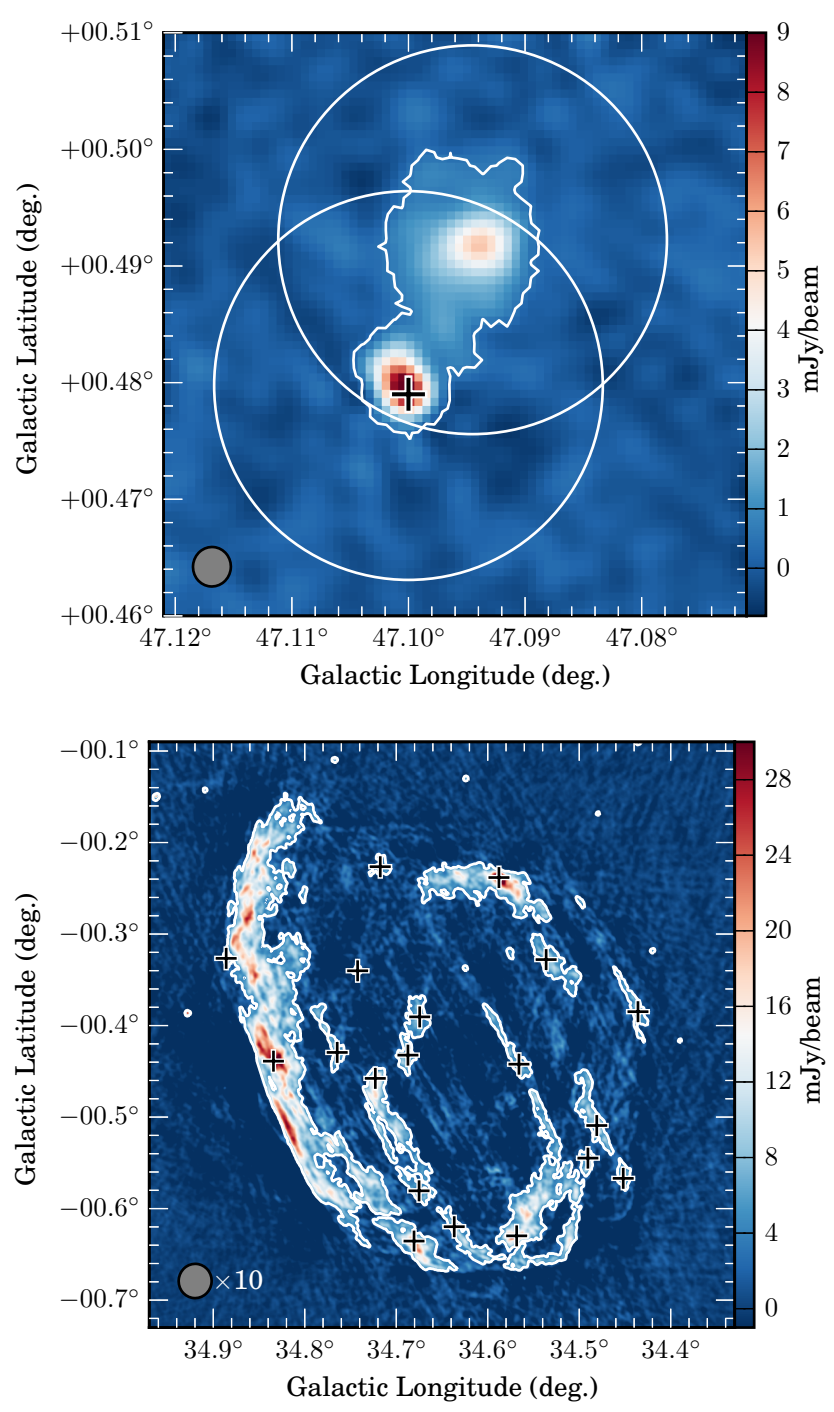

Fig. 5. Examples of source identification in the catalog. In both panels, the white contours represent the area of the source extracted by BLOBCAT overlaid on the averaged image of spw-1440 and spw-1820, and the crosses denote the sources in the catalog. Top panel: source G47.100+0.479 consists of $2 \mathrm{H}$ II regions (indicated with white circles, Anderson et al. 2014) close together. Bottom panel: the SNR W44 (e.g., Green 2014) is resolved into 19 sources in our catalog. The synthesized beam is shown in the bottom left corner of each panel. The beam in the right panel is scaled up to 10 times its original size.

With a matching radius of $5^{\prime \prime}$, we found 1320 unresolved THOR sources matched with the NRAO VLA Sky Survey (NVSS; Condon et al. 1998) sources. We compared the $1.4 \mathrm{GHz}$ flux densities from THOR and NVSS; the THOR flux at spw1440 is also tightly correlated with, but slightly higher than, the flux from NVSS. The median value of the NVSS/THOR flux ratio is about 0.93 . By comparing the THOR 1.4 and $1.8 \mathrm{GHz}$ average flux density of the first half of the survey with the NVSS flux density, Bihr et al. (2016) found similar results (see also Fig. 17 in Bihr et al. 2016). This discrepancy is yet undetermined, but this is beyond the scope of this work. The NVSS images show that the NVSS catalog is severely contaminated with obvious false detections, which could be due to side lobes from strong sources close to the Galactic plane or ghost artifacts (Grobler et al. 2014).

For the second half of the THOR survey, we applied the same data reduction method and source extraction algorithm as the first
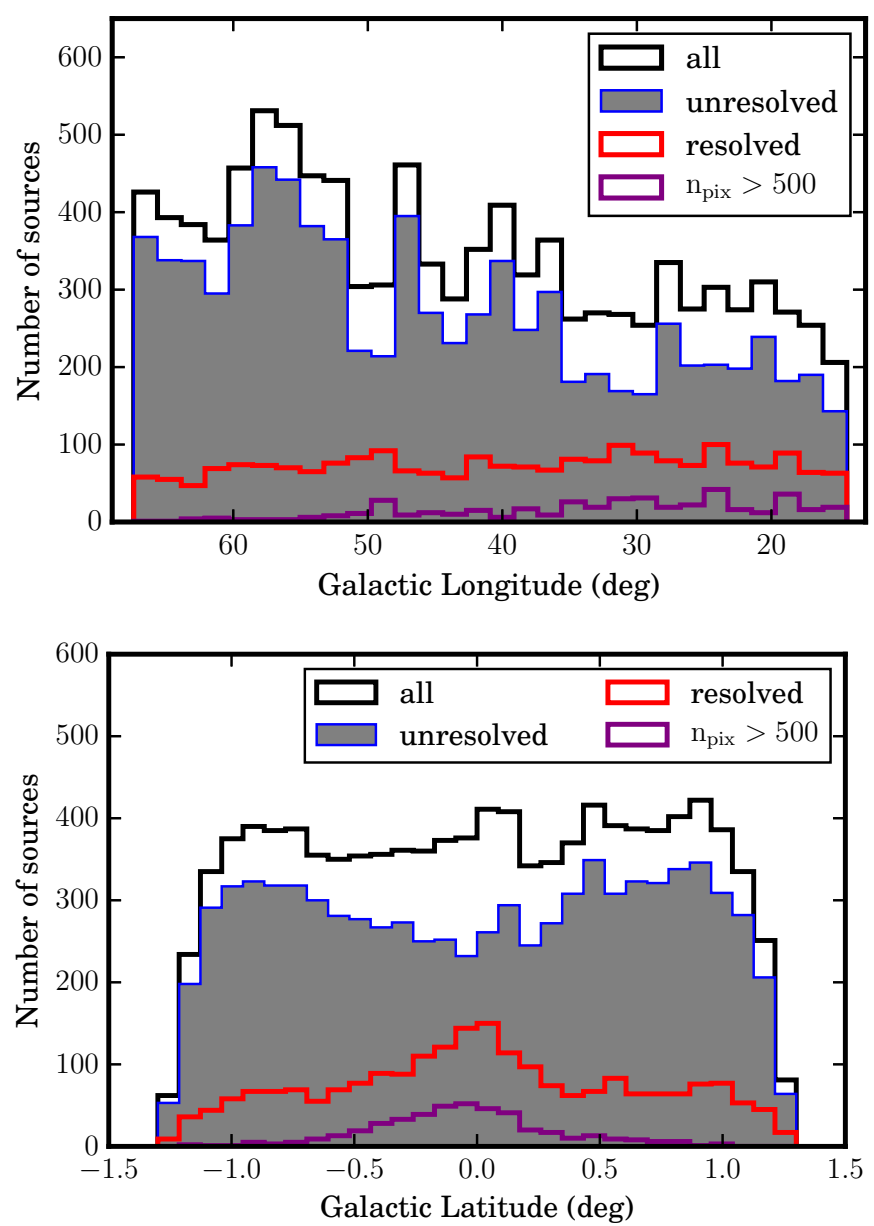

Fig. 6. Histograms for the source distribution along Galactic longitude (top panel) and latitude (bottom panel). In both panels, the black histogram shows the distribution of all the sources in the catalog; the other histograms show percentage of the catalog as indicated in each panel.

half (Bihr et al. 2016). Since there are overlapping areas between the first and second half of the survey, we further compare the position and flux density of the common sources between the two halves.Of the sources, 94\% have position differences $<2.5^{\prime \prime}$ (the pixel size of the maps) and $93 \%$ of the sources have flux differences $<1 \mathrm{mJy}$ beam $^{-1}$. Therefore, the second half of the survey is consistent with the first half and with the previous radio surveys.

To verify the reliability of our spectral index determination, we extrapolated the flux density of selected sources to $5 \mathrm{GHz}$ and compared this with the CORNISH catalog. The CORNISH cata$\log$ contains 2493 sources within the THOR survey area. With a matching radius of $5^{\prime \prime}$, we found 1905 matches between THOR and CORNISH. We selected unresolved sources with a THOR spectral index between 0 and -0.2 (optically thin free-free emission has a spectral index -0.1), which are detected in all six SPWs in THOR (fit_spws $=6$ ). We selected 68 sources this way. We estimated the flux density at $5 \mathrm{GHz}$ of these 68 sources according to their spectral indices from the THOR catalog. The extrapolated flux densities are very close to the flux densities in the CORNISH catalog as shown in Fig. 8. Sources with a spectral index measurement of between -0.09 and -0.11 (red in Fig. 8) are closer to ideal optically thin, and the extrapolated flux for these sources also agree better with those from CORNISH as expected. 


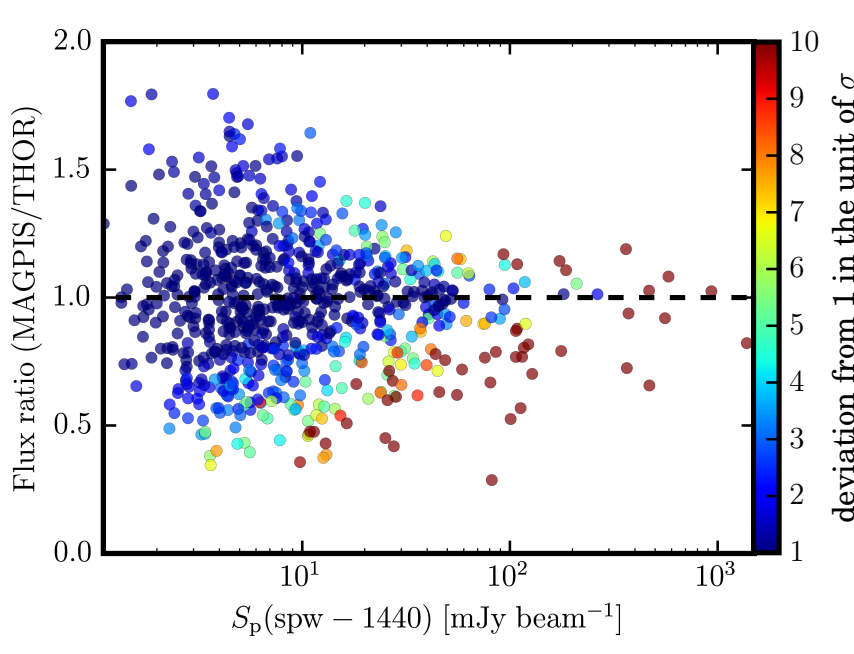

Fig. 7. Ratio of the MAGPIS and THOR flux density plotted against the THOR flux density. The points are colored according to their deviation from a flux ratio of unity in units of the uncertainty in the ratio, so the deviation $=(1-$ ratio $) / \sigma_{\text {ratio }}$. The dashed line represents a one-to-one relation.

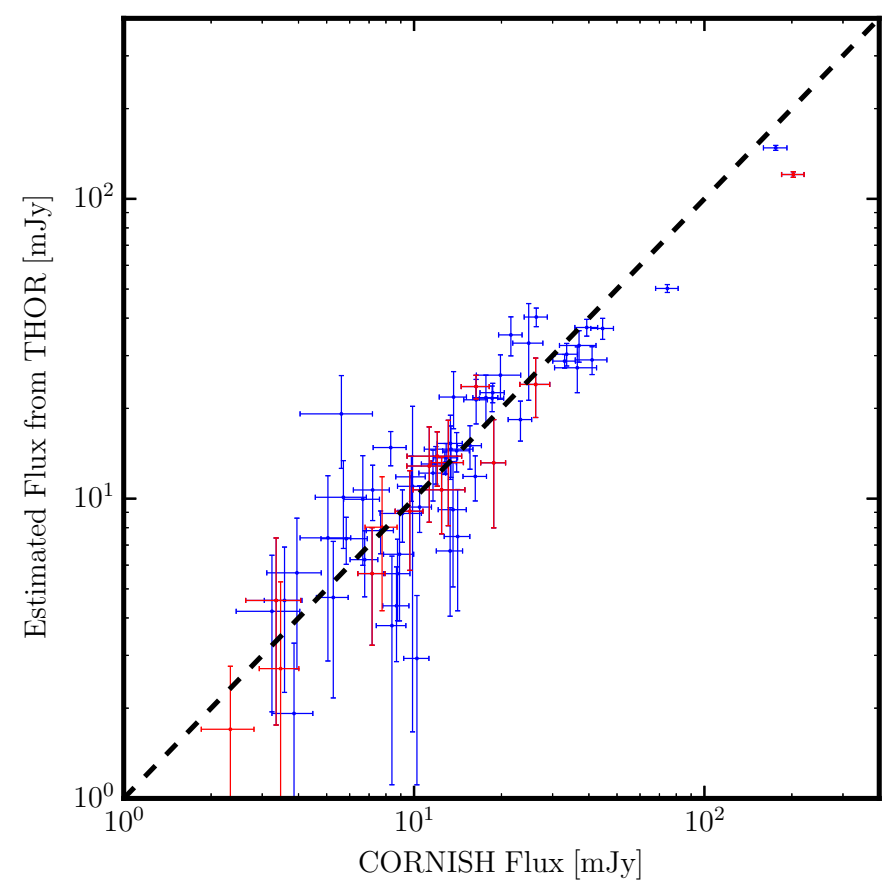

Fig. 8. Extrapolated flux densities at $5 \mathrm{GHz}$ for selected sources according to their spectral indices from the THOR catalog plotted against the flux densities in the CORNISH catalog. Sources with a spectral index between 0 and -0.2 are shown in blue, and those with a spectral index -0.09 and -0.11 are shown in red. The dashed line represents a one-toone relation.

Furthermore, we also compared the THOR spectral indices with spectral indices Kalcheva et al. (2018) derived for the CORNISH UCH II regions. Kalcheva et al. (2018) used flux density measurements of CORNISH $5 \mathrm{GHz}$ and MAGPIS $20 \mathrm{~cm}$ to derive the spectral indices. Eleven unresolved THOR sources are associated with the CORNISH UCH II regions and have a reliable spectral index measurement in Kalcheva et al. (2018). The comparison again reveals that the spectral indices from these two studies are tightly correlated.

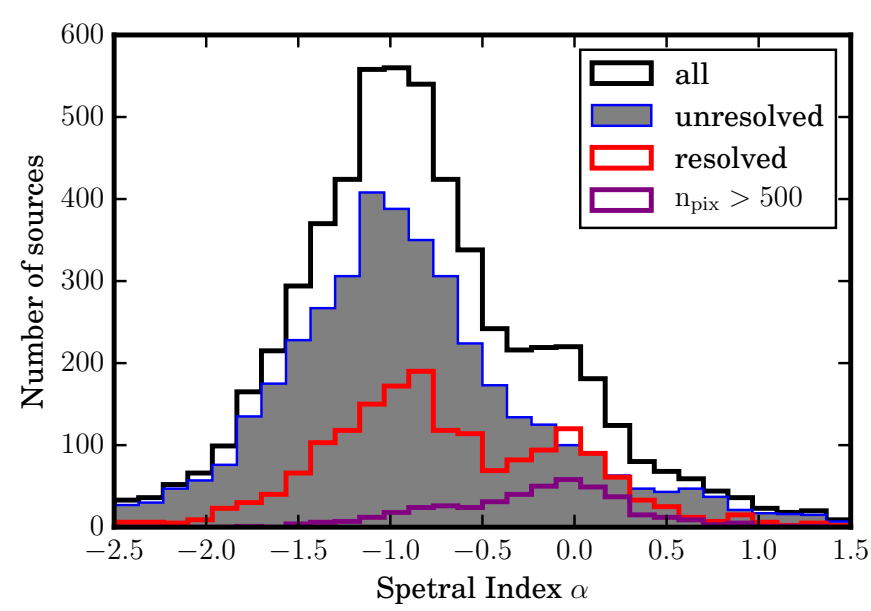

Fig. 9. Histogram of the spectral index for all reliably detected sources (fit_spws $\geq 4)$ with a reliable spectral index measurement $(\sim 5857$ sources).

\subsection{Spectral index}

As described in Sect. 3, we derived spectral indices for 8228 sources, of which we consider the values for 5857 sources as reliable (fit_spws $\geq 4$ ). Using the spectral index information we can distinguish the physical origin of the emission and classify the continuum sources. Figure 9 shows the distribution of the spectral indices for all detected sources with a reliable spectral index measurement. For all sources the spectral index distribution shows a strong peak around $\alpha \sim-1$, and a secondary peak around $\alpha \sim 0$. If we consider only the unresolved sources, the spectral index distribution peaks around $\alpha \sim-1$, and the secondary peak around $\alpha \sim 0$ diminishes. Most of the unresolved sources show a negative spectral index indicating that they are dominated by non-thermal synchrotron radiation. Considering the unresolved sources are also evenly distributed along Galactic longitude and latitude (Sect. 4), these unresolved sources with a negative spectral index are likely to be mostly extragalactic sources. The spectral distribution of the resolved sources shows two clear peaks around $\alpha \sim-1$ and $\alpha \sim 0$. Most of the resolved sources with a flat or positive spectral index are Galactic H II regions (see Sect. 5.3). Those with negative spectral index are mostly SNRs (see Sect. 5.4) and radio galaxies (see Sect. 5.8), or overlapping unresolved sources classified as one single source. Considering that only the very large sources that have an area in pixels larger than $500\left(n_{\text {pix }}>500\right.$, effective radius $\left.\gtrsim 32^{\prime \prime}\right)$, the spectral index distribution peaks around $\alpha \sim 0$ and extends to $\alpha \sim-1$. Among these large sources, about $80 \%$ are associated with $\mathrm{H}_{\mathrm{II}}$ regions and the rest are classified as SNRs and radio galaxies (see Sect. 5.3, 5.4, and 5.8).

\subsection{H II regions}

Using data from the all-sky Wide-Field infrared Survey Explorer (WISE) satellite, Anderson et al. (2014) made the most complete catalog of $\mathrm{H}_{\text {II }}$ regions to date with a total of more than 8000 sources ${ }^{10}$. Within the region of the THOR survey, the WISE H II catalog contains $\sim 2400$ sources, and $\sim 1500$ of them show radio continuum emission. The size of the $\mathrm{H}$ II regions varies from $10^{\prime \prime}$ to $20^{\prime}$ from the mid-infrared (MIR) images, so multiple Galactic and extragalactic sources could easily be enclosed within one

$\overline{10}$ http://astro.phys.wvu. edu/wise/ 
single large H in region. Bihr et al. (2016) showed that matching the THOR continuum sources with the WISE catalog sources with $r<150^{\prime \prime}$ in an automated fashion would produce less than $10 \%$ false matches. We take the same radius threshold when doing the automated matching, then we visually inspect the results and remove the false detections. We further visually compare the remaining large WISE sources $\left(r>150^{\prime \prime}\right)$ with our continuum images, and identify the continuum sources that are associated with $\mathrm{H}$ II regions.

In addition to the WISE catalog, $239 \mathrm{UCH}$ II regions are identified in the CORNISH survey (Kalcheva et al. 2018) and 205 lie within the THOR coverage. With a matching radius of the effective radius equal to each THOR continuum source, 202 UCH II regions have counterparts in the THOR survey. For sensitivity reasons, we do not detect three CORNISH $\mathrm{UCH}$ II regions (G024.1839+00.1199, G026.1094-00.0937, and G030.0096-00.2734; see Kalcheva et al. 2018). All the continuum sources that are associated with UCH II regions are also associated with at least one $\mathrm{H}_{\mathrm{II}}$ region in the WISE catalog except G26.008+0.137 and G37.735-0.113; therefore we list the CORNISH UCH II region counterparts for only these two continuum sources.

In total, we matched 713 continuum sources with $\mathrm{H}$ II regions. Among the matched $\mathrm{H}$ II regions, 16 are in the radio quiet group in the WISE catalog, which means no radio continuum emission is detected in the MAGPIS (Helfand et al. 2006) and VGPS (Stil et al. 2006) surveys. H in regions close to each other could be identified as one single source in our catalog (left panel, Fig. 5). In total 231 continuum sources are matched with more than one $\mathrm{H}$ II region. In particular, G43.171+0.007 is matched with $19 \mathrm{H} \mathrm{II}$ regions in W49, G19.610-0.235 is matched with $7 \mathrm{H}$ II regions, G45.122+0.132 encompasses 6 H II regions, G48.610+0.027 encompasses $9 \mathrm{H}$ II regions, G49.370-0.302 encompasses $8 \mathrm{H}$ II regions (W51), and G49.488-0.380 encompasses $11 \mathrm{H}$ II regions (W51). The radius of the matched $\mathrm{H}_{\text {II }}$ regions measured at MIR in the WISE catalog varies from $12^{\prime \prime}$ to $20^{\prime}$ with a median value of $\sim 60^{\prime \prime}$.

We show in Fig. 10 one of the extreme cases, G43.171+0.007, which is associated with $19 \mathrm{H}$ II regions in W49. By comparing ATLASGAL and CORNISH surveys, Urquhart et al. (2013) identified $18 \mathrm{UCH}$ II regions associated with this region and found that this region has the highest UCH II surface density in the first quadrant. The spectral index map of W49 (Fig. 10) reveals a positive or flat spectral index toward most of the area of the source, except the edge regions, where the spectral index fitting is not reliable owingto low $\mathrm{S} / \mathrm{N}$. This indicates that the $\mathrm{H}$ in region is dominated by thermal free-free emission. The spectral indices toward some strong continuum peaks are even larger than 0.5 ; this could indicate optically thick free-free emission.

To derive the physical properties of the $\mathrm{H}$ in regions, we fit the spectral energy distribution (SED) of the $\mathrm{H}$ II region sources with a simple homogeneous $\mathrm{H}$ in region model with optical depth to get the emission measure (EM) of the $\mathrm{H}_{\text {II }}$ regions (Sánchez-Monge 2011). We selected continuum sources with a $\mathrm{S} / \mathrm{N}$ larger than $7 \sigma$, fit_spws $=6$ (see Sect. 5.2), and spectral index $\alpha>-0.3$. We further limited our fitting sample to sources with an effective radius smaller than $60^{\prime \prime}$, since tests performed by Bihr et al. (2016) have shown that we are able to recover sources with size up to $\sim 120^{\prime \prime}$ reasonably well (80\% flux recovery). Among 713 continuum sources that are matched with $\mathrm{H}_{\text {II }}$ regions, 262 match our selection criteria and are fitted. We fit the peak flux of the sources assuming that the line-of-sight size of the sources are the same as the beam size $\left(25^{\prime \prime}\right)$; this allows us to get the EM and the electron density $\left(n_{\mathrm{e}}\right)$ if the distance information is available

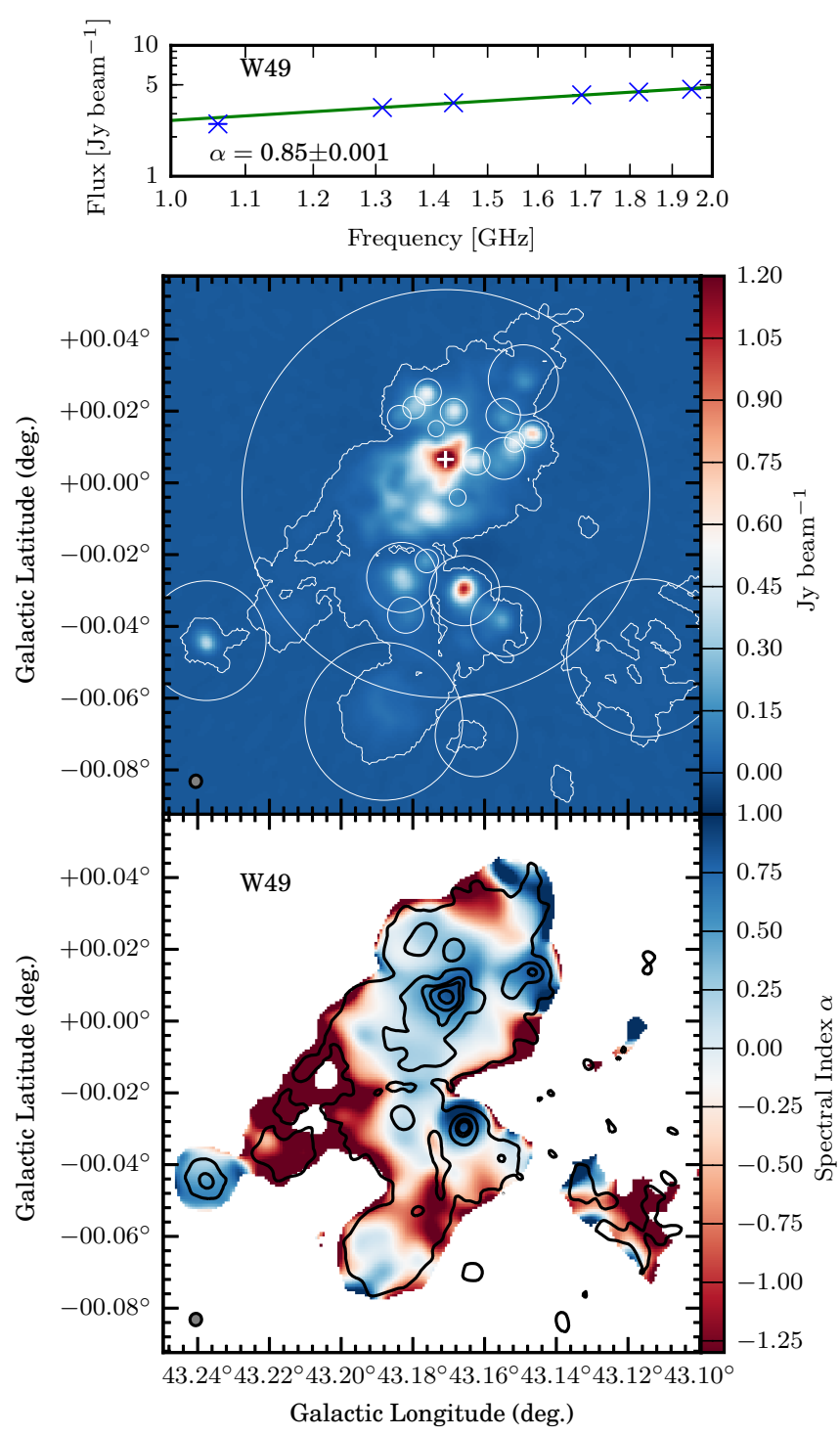

Fig. 10. Spectral index map for the $\mathrm{H}_{\mathrm{II}}$ region W49. Top panel: spectral index fitting result of the peak of the continuum source G43.171+0.007 (denoted with a cross in the middle panel). Middle panel: averaged image of spw-1440 and spw-1820, the white contours represent the area of the source extracted by BLOBCAT, and the cross denotes the peak of the source G43.171+0.007 in the catalog. The circles indicate the $\mathrm{H}$ II regions from Anderson et al. (2014) matched with the continuum sources in the THOR catalog. Bottom panel: the spectral index map produced by fitting the flux of the six SPWs pixel by pixel. The synthesized beams of the averaged image and the spectral index map are shown in the bottom left corner of each panel.

in the WISE catalog (Anderson et al. 2014). For 168 sources we got a good fit to the model and list the fitting results in Table 4 . The main uncertainties of the fitting results are from the uncertainties of the peak flux brought in by the flux calibrator $(\sim 5 \%$ at these wavelengths), uncertainties from the fitting procedure $(<10 \%)$, and the uncertainties of the sizes of the sources on the line of sight. Two sources have $T_{\mathrm{e}}$ measurements from previous RRLs observations (Balser et al. 2011), and we assume a $T_{\mathrm{e}}$ of $8000 \mathrm{~K}$ for the remaining sources; the mean $T_{\mathrm{e}}$ estimated by Balser et al. (2011) is $\sim 8000 \mathrm{~K}$ for $\mathrm{H}$ in regions in the THOR survey area. For 151 sources we fitted an $10^{4}<\mathrm{EM}<10^{5} \mathrm{~cm}^{-6}$ pc. For 124 sources there is distance information in the WISE catalog, thus we estimated $n_{\mathrm{e}}$, which is between $0.9 \times 10^{2}$ and $5.4 \times 10^{2} \mathrm{~cm}^{-3}$. 
Table 4. Fitting results for $\mathrm{H}_{\text {II }}$ regions.

\begin{tabular}{lccccccc}
\hline \hline Gal.ID & $\alpha$ & $\Delta \alpha$ & H II name & $\begin{array}{c}T_{\mathrm{e}}{ }^{a} \\
(\mathrm{~K})\end{array}$ & $\begin{array}{c}d^{b} \\
(\mathrm{kpc})\end{array}$ & $\begin{array}{c}\mathrm{EM} \\
\left(\times 10^{5} \mathrm{~cm}^{-6} \mathrm{pc}\right)\end{array}$ & $\begin{array}{c}n_{\mathrm{e}}{ }^{b} \\
\left(\times 10^{2} \mathrm{~cm}^{-3}\right)\end{array}$ \\
\hline G52.753+0.334 & 0.13 & 0.01 & G052.750+00.334 & 8970 & 9.575 & 2.0 & 5.0 \\
G53.187+0.209 & -0.14 & 0.02 & $\mathrm{G} 053.188+00.209$ & - & 9.96 & 0.7 & 2.9 \\
G56.160+0.077 & -0.21 & 0.04 & $\mathrm{G} 056.153+00.076$ & - & 9.9 & 0.2 & 1.7 \\
G56.420+0.423 & -0.12 & 0.06 & $\mathrm{G} 056.412+00.423$ & - & 9.93 & 0.2 & 1.6 \\
G57.547-0.272 & -0.05 & 0.01 & $\mathrm{G} 057.541-00.279$ & - & 8.805 & 1.3 & 4.2 \\
G58.773+0.646 & -0.10 & 0.04 & $\mathrm{G} 058.769+00.648$ & - & 4.405 & 0.2 & 2.5 \\
G60.883-0.130 & 0.01 & 0.02 & G060.881-00.135 & 7463 & - & 1.4 & - \\
\hline
\end{tabular}

Notes. ${ }^{(a)} T_{\mathrm{e}}$ is assumed to be $8000 \mathrm{~K}$ if it is not available from the WISE catalog. ${ }^{(b)}$ We calculate $n_{\mathrm{e}}$ if distance is available from the WISE catalog. The full table is available at the CDS.

Continuum source G49.477-0.328, which is associated with the $\mathrm{H}$ II region G049.490-00.381 in W51, has the highest value for EM and $n_{\mathrm{e}}$ from our fitting, which are $4.2 \times 10^{5} \mathrm{~cm}^{-6} \mathrm{pc}$ and $3.1 \times 10^{4} \mathrm{~cm}^{-3}$, respectively.

Among the 168 sources for which we can get a good H II region model fit, 39 are also associated with CORNISH UCH II regions. Assuming homogeneous optically thin and the line-ofsight size is the same as the angular size, Kalcheva et al. (2018) calculated EM and $n_{\mathrm{e}}$ for the CORNISH UCH II regions. Since CORNISH is at $5 \mathrm{GHz}$ and the angular sizes of the $39 \mathrm{UCH}$ II regions they derived are all smaller than $12^{\prime \prime}$, which is about a half beam size of THOR, we cannot compare the EM and $n_{\mathrm{e}}$ from the the two samples directly. If we assume all $39 \mathrm{UCH}$ in regions have a size of $25^{\prime \prime}$, and convert the EM and $n_{\mathrm{e}}$ from the CORNISH observations accordingly, these values are close to those derived with the THOR observations; however they have a large scatter. We can fit the $\mathrm{EM}_{\mathrm{CORNISH}}$ versus $\mathrm{EM}_{\mathrm{THOR}}$ and $n_{\mathrm{eCORNISH}}$ versus $n_{\text {eTHOR }}$ plots with a linear function $y=x * 0.8+c$. This is a very rough comparison, but shows our $\mathrm{H}$ II fitting procedure is correct.

\subsection{Supernova remnants}

Green (2014) provided the most complete catalog of Galactic SNRs with 294 sources, 67 of which lie, or partially lie, in our survey area. By visually comparing the catalog with our continuum images, we identified 92 continuum sources associated with 36 SNRs. As shown in Fig. 5, many of the SNRs are resolved into multiple sources by the source-finding algorithm. The rest of the SNRs from Green (2014) either have too weak radio continuum emission, which is below our sensitivity, or are too diffuse and are filtered out by the VLA C-configuration (see also Bihr et al. 2016). We marked all the matched continuum sources with "SNR_green" in the catalog and list the corresponding ID of the SNRs from Green (2014).

By studying the compact and extended THOR+VGPS $1.4 \mathrm{GHz}$ continuum emission (in the region $l>17.5^{\circ}$ ), Anderson et al. (2017) confirmed the radio emission for 52 SNRs from Green (2014). Anderson et al. (2017) found that 6 of the SNRs from the Green (2014) catalog (G20.4+0.1, G21.5 0.1, G23.6+0.3, G54.1+0.3, G59.8+1.2, and G065.8 0.5) are confused with $\mathrm{H}_{\text {II }}$ regions and the radio emission appears to be thermal. Figure 11 shows the spectral index fitting result and the spectral index map of SNR W49B as an example, indicating that the region is dominated by negative spectral index with some variation, and further indicating non-thermal synchrotron emission.
By comparing the large scale diffuse radio continuum emission traced by the combined THOR+VGPS $1.4 \mathrm{GHz}$ continuum data with the MIR Spitzer GLIMPSE $8.0 \mu \mathrm{m}$ (Benjamin et al. 2003; Churchwell et al. 2009) and MIPSGAL $24 \mu \mathrm{m}$ (Carey et al. 2009) data, Anderson et al. (2017) identified 76 new SNR candidates. Since the radio continuum emission from most of the new SNR candidates is weak and diffuse, we could only detect 13 of these candidates in our continuum catalog, which uses only THOR C-configuration data, and hence do not trace the large scale diffuse emission. Among the new SNR candidates, G17.80-0.02, G26.75+0.73, and G27.06+0.04 are matched with 2 continuum sources each in our catalog, and G51.21+0.11 is matched with 6 continuum sources. We marked all the matched continuum sources with "SNR_anderson" in the catalog and list the corresponding ID of the SNRs from Anderson et al. (2017).

The MAGPIS survey (Helfand et al. 2006) identified many SNR candidates, and 33 of these are covered by our THOR survey. Anderson et al. (2017) found that 17 MAGPIS SNR candidates (G18.2536-0.3083, G19.4611+0.1444, G19.5800-0.2400, G19.5917+0.0250, G19.6100-0.1200, G19.6600-0.2200, G21.6417+0.0000, G22.7583-0.49171, G22.9917-0.3583, G23.5667-0.0333, G24.1803+0.2167, G25.2222+0.2917, G29.0667-0.6750, G30.8486+0.1333, $\mathrm{G} 31.0583+0.4833, \mathrm{G} 31.6097+0.3347$, and G31.8208-0.1222.) are spatially coincident with a known $\mathrm{H}_{\text {II }}$ region from the WISE catalog. G18.2536-0.3083 is also reported as a known H II region by Bihr et al. (2016). Furthermore, G16.3583-0.1833 and G17.3361-0.1389 are spatially coincident with known $\mathrm{H}_{\text {II }}$ regions G016.360-00.211 and G017.336-00.146 from the WISE catalog. G29.0778+0.4542 is a known PN (PNG029.0+00.4; see also Anderson et al. 2017). For 10 MAGPIS SNRs (Helfand et al. 2006) we found matched continuum sources in our catalog, however, these continuum sources are also matched with SNRs from Green (2014) or candidates from Anderson et al. (2017) and we do not mark them separately in the catalog. For 3 MAGPIS SNRs we do not find matched continuum sources owing to THOR sensitivity and the missing flux problem (see also Bihr et al. 2016).

\subsection{Planetary nebulae}

The Hong Kong/AAO/Strasbourg $\mathrm{H} \alpha$ PN database (HASH, Parker et al. 2016) provides up-to date information for all known Galactic PNe. In the HASH database, there are $234 \mathrm{PNe}$ with the PN status marked as T (True PN), L (Likely PN), and P (Possible PN) within our survey area. We detected 164 in our catalog, among which 75 are true PN, 60 are likely PN, and 29 are possible 
PN. We marked them as "PN" in our catalog, and list the HASH ID (PNG). In particular PNG029.2+00.0 is associated with the continuum source G29.211-0.069, the extended emission traces the known $\mathrm{H}_{\text {II }}$ region G029.165-00.035. Thus we indicate the continuum source G29.211-0.069 as "HII;PN" and list both the PN and H II IDs. Furthermore, 90 of the matched PNe do not have radio emission information at $20 \mathrm{~cm}$ in the database; our survey provides this important information. We list all the PNe detected in the THOR survey in Table D.1. For the remaining 74 sources the peak flux at spw-1440 from THOR observations agree with the radio flux at $20 \mathrm{~cm}$ from the database in general and have a median flux difference of $31 \%$ relative to those in the HASH database. Since we are comparing flux density in the HASH database with the THOR peak flux, the large difference is expected.

In addition to the 164 detected $\mathrm{PNe}$ we include in our catalog, PNG 014.5+00.0, 017.4+00.3, and 018.7+00.0 are also detected in our observations. However, the continuum sources that these $\mathrm{PNe}$ are associated with are known $\mathrm{H}_{\text {II }}$ regions (G014.598+00.019, G017.414+00.377, and G018.710+00.000). Since these $3 \mathrm{PNe}$ are all possible PN, we did not include them in our catalog.

We did not detect the remaining 67 PNe, most likely for sensitivity reasons. Only 4 of the $67 \mathrm{PNe}$ have $1.4 \mathrm{GHz}$ radio flux information in the HASH catalog. The $1.4 \mathrm{GHz}$ flux density of PNG029.8+00.5 in the HASH catalog is $33 \mathrm{mJy}$, however, we did not detect any radio counterpart in our THOR-only nor THOR+VGPS data. Bojičić et al. (2011) and Luo et al. (2005) also reported the radio detection for this $\mathrm{PN}$ as suspect. The $1.4 \mathrm{GHz}$ flux of PNG028.7+00.7, 044.9+00.0, and 056.1-00.4 in the HASH catalog is $2.7,3.5$, and $6.6 \mathrm{mJy}$, respectively.

\subsection{Pulsars}

The ATNF Pulsar Catalogue ${ }^{11}$ (Manchester et al. 2005) provides the most complete catalog of all published rotation-powered pulsars. Within our survey region, the catalog contains 335 pulsars. We cross-matched the pulsar catalog with the THOR continuum catalog taking into account the position uncertainty from the pulsar catalog. We matched 38 THOR continuum sources with pulsars, denoted these as "PSR" in the continuum catalog, and list the corresponding pulsar names. Except for G38.163-0.151, all matches have a separation smaller than $7.5^{\prime \prime}$. Considering the synthesized beam size of our observation is $\sim 12^{\prime \prime}-18^{\prime \prime}$, these matches are reliable. G38.163-0.151 is matched with J1901+0435 with a separation of $15^{\prime \prime}$, however, $\mathrm{J} 1901+0435$ has a relatively large position uncertainty of $10^{\prime \prime}$, which we consider a good match. We list the matched continuum sources and pulsars in Table E.1.

Among all the pulsars detected in our survey, J1841-0500 is an extremely intermittent pulsar (Camilo et al. 2012) and has no $1.4 \mathrm{GHz}$ radio flux information in the ATNF pulsar catalog. We detected a radio counterpart in our observation with a $S / N \sim 6$ (G27.322-0.033).

We did not detect the remaining 297 pulsars in our catalog, which is mainly because of the low sensitivity in some sections of the maps. Among the non-detected pulsars, 247 have mean $1.4 \mathrm{GHz}$ radio flux densities in the ATNF pulsar catalog, and their mean flux densities are all below $3 \mathrm{mJy}$, with a median value of $0.3 \mathrm{mJy}$. In comparison, the detected pulsars have a median mean flux density of $1.7 \mathrm{mJy}$ from the ATNF catalog.

\footnotetext{
11 http://www.atnf.csiro.au/research/pulsar/psrcat
}
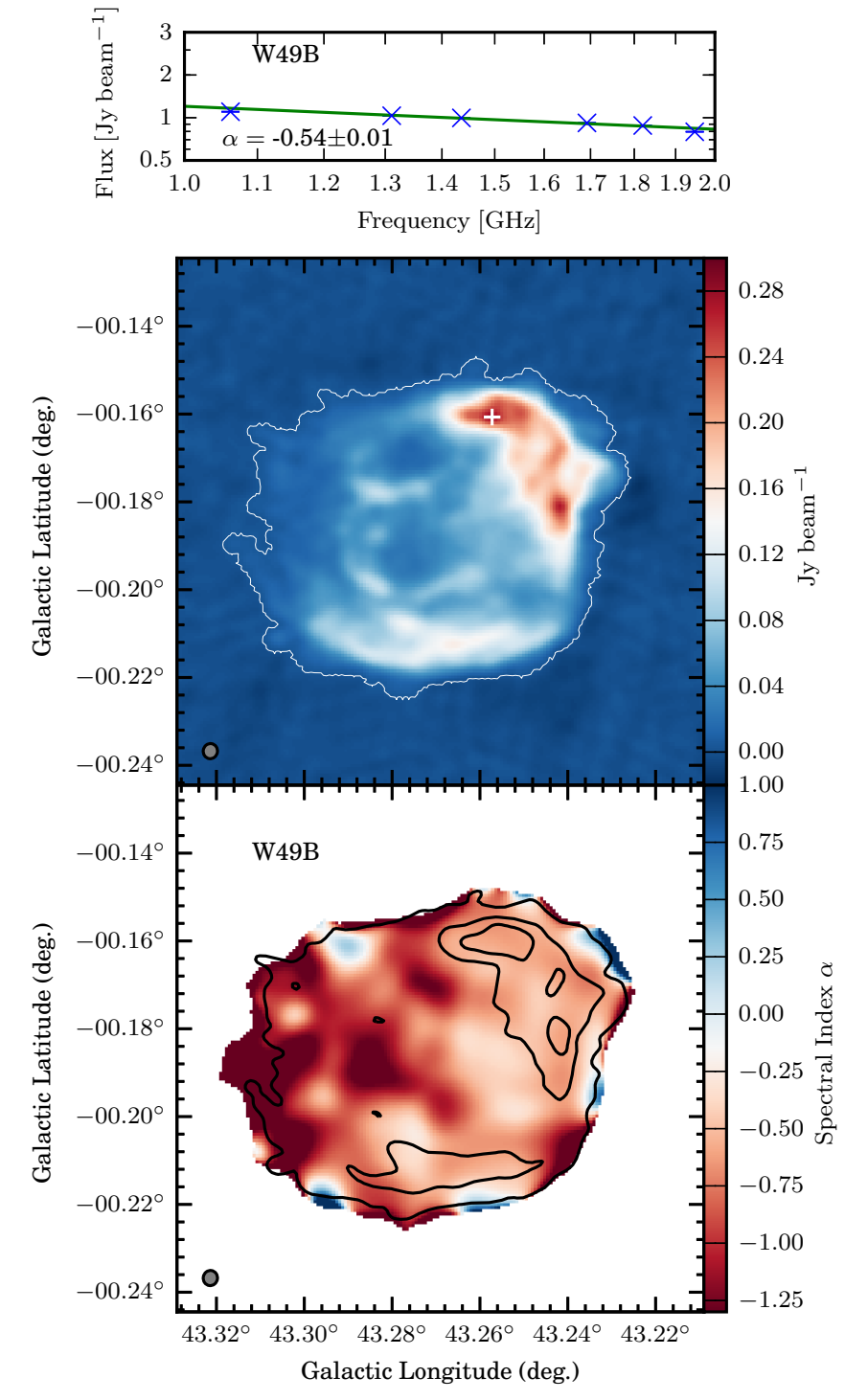

Fig. 11. Spectral index map for SNR W49B. Top panel: spectral index fitting result of the peak of the continuum source G43.257-0.161. Middle panel: averaged image of spw-1440 and spw-1820, the white contours represent the area of the source extracted by BLOBCAT, and the cross denotes the peak of the source G43.257-0.161 in the catalog. Bottom panel: the spectral index map produced by fitting the flux of the 6 SPWs pixel by pixel. The synthesized beams of the averaged image and the spectral index map are shown in the bottom left corner of each panel.

In general, the peak flux at spw-1440 from THOR observations are in agreement with the radio flux at $1.4 \mathrm{GHz}$ in the ATNF catalog and have a median flux difference of $30 \%$ relative to those in the ATNF catalog.

\subsection{X-ray sources}

While diffuse radio and $\mathrm{X}$-ray emission can trace $\mathrm{H}_{\text {II }}$ regions (free-free emission and stellar wind shocks; Silich et al. 2005) and SNRs (e.g., Decourchelle et al. 2005), the compact emission usually traces pulsar wind nebulae (e.g., Brisken et al. 2005; Miller et al. 2005), XRBs or microquasars (Mirabel \& Rodríguez 1998, 1999), or AGNs (e.g, Bridle \& Perley 1984; Harris \& Krawczynski 2006). 

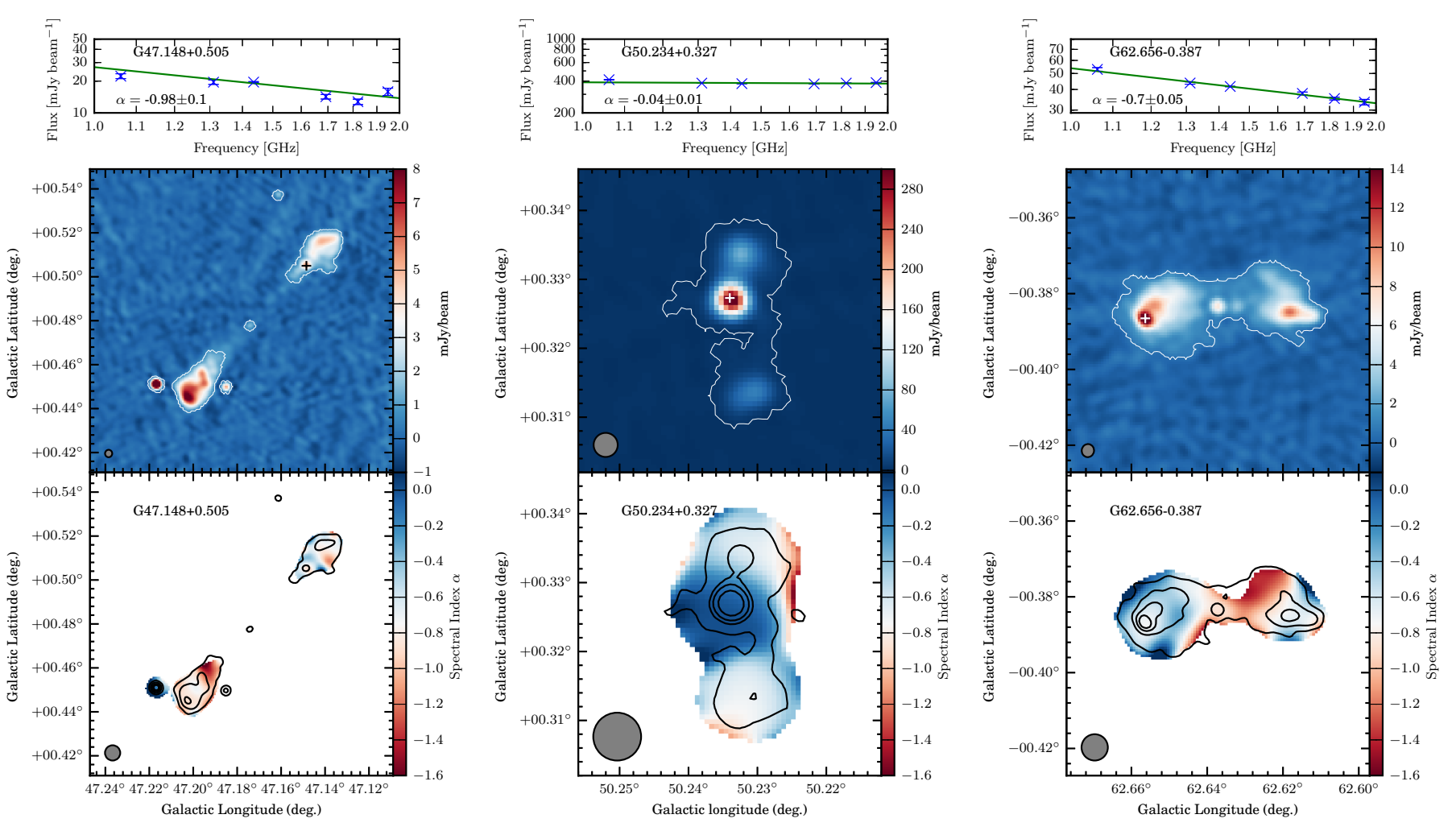

Fig. 12. Spectral index maps for three "jet" sources. Top panels: spectral indices fitting results of the peak positions. Middle panels: averaged images of spw-1440 and spw-1820, the white contours represent the area of the sources extracted by BLOBCAT, and the crosses denote the peaks of the sources in the catalog. Bottom panels: the spectral index map produced by fitting the flux of the 6 SPWs pixel by pixel. The synthesized beams of the averaged image and spectral index map are shown in the bottom left corner of each panel.

We cross-matched our continuum catalog with three Xray source catalogs as follows: 1SXPS SWIFT X-ray telescope point source catalog (Evans et al. 2014), XMM-Newton Serendipitous Source Catalog (3XMM-DR7 Version; Watson et al. 2009; Rosen et al. 2016), and Chandra Source Catalog (CSC, v1.1; Evans et al. 2010). Within our survey area, 1SXPS has $\sim 2800$ sources, CSC has $\sim 3900$ sources, and $3 \mathrm{XMM}$ has $\sim 11500$ sources. With a matching radius of $10^{\prime \prime}$, we found that 79 of the remaining $\sim 9300$ THOR sources are associated with X-ray sources (see Table F.1). Among the $79 \mathrm{X}$-ray sources we detected, 43 do not have a radio counterpart within a radius of $15^{\prime \prime}$ in the SIMBAD Astronomical Database ${ }^{12}$ or the NASA/IPAC Extragalactic Database (NED) ${ }^{13}$. Sixty-five of the X-ray sources are unresolved and the remaining resolved sources are also relatively compact with an effective radius $<29^{\prime \prime}$. In particular, continuum source G45.366-0.219 is associated with the microquasar GRS1915+105 (Mirabel \& Rodríguez 1999). Further H I absorption studies toward these sources would allow us to identify which ones are Galactic. By investigating the $\mathrm{X}$-ray and radio flux ratios, the spectral indices and observations at other (optical/infrared) wavelengths of the Galactic sources in detail, we can further constrain the type of the sources, i.e., low mass XRB, PN, pulsar etc. (e.g., Seaquist 1993; Maccarone et al. 2012; Tetarenko et al. 2016).

\footnotetext{
12 http://simbad.u-strasbg.fr/simbad/

13 https://ned.ipac.caltech.edu/
}

\subsection{Extragalactic radio sources}

About 9300 sources in our catalog are not classified. Taking a matching radius of $15^{\prime \prime}$, which is the average size of the synthesized beam of the averaged images used for source extraction, more than 7750 sources do not match to anything in SIMBAD. For the matched sources, about $84 \%$ are matched to only one or more radio sources. With the same matching radius $\left(15^{\prime \prime}\right)$ in the NED database, $\sim 3350$ of the sources are matched to one or more infrared sources, $\sim 2140$ of the sources are matched to one or more radio sources, and more than 3760 sources do not have any counterparts within a radius of $15^{\prime \prime}$. This means that for 9000 sources detected in our survey, we do not know exactly what they are other than that they have radio and/or infrared emission.

As we mentioned in Sect. 5.2, aside from the $\mathrm{H}_{\text {II }}$ regions, SNRs, and PNe, the majority of the sources we detected are actually extragalactic. Many of the those sources are also resolved and show bipolar radio lobe structures. We checked all the resolved sources with a negative or flat spectral index in our catalog by eye, identified $\sim 300$ sources that show clear bipolar jet structure, and marked these as "jet" in the catalog. For these jet sources, we can construct the spectral index maps, which could be used to estimate the source expansion velocity when combined with magnetic field strength information. Since the identification of these jet sources is done morphologically, two partially overlapped sources could also be categorised into jet sources. Since we do not have zero-spacing information, we filtered out large scale structures in our observations. Bihr et al. (2016) showed that the THOR observations are able to recover sources with sizes up to $\sim 120^{\prime \prime}$ reasonably well ( $\geq 80 \%$ flux recovery); 

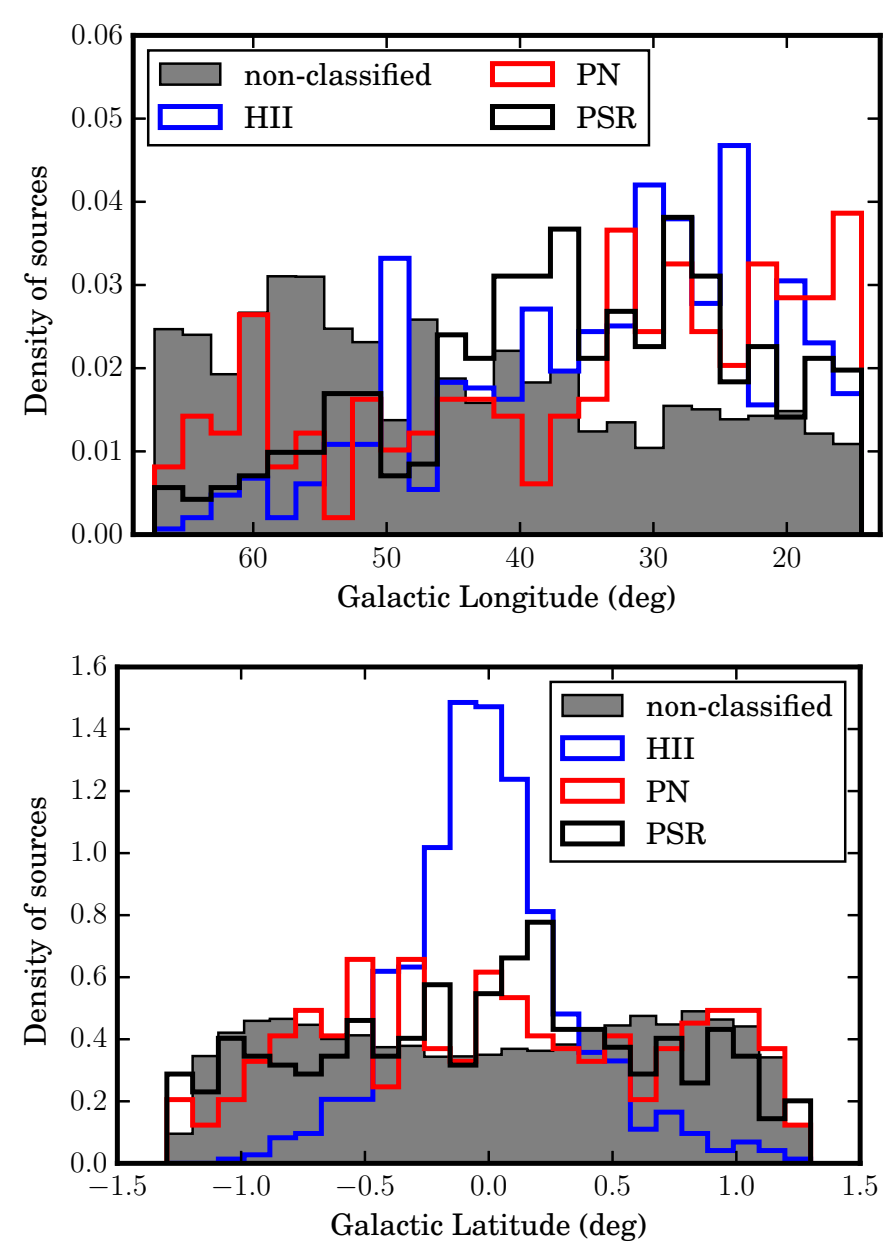

Fig. 13. Normalized distribution of different types of objects along Galactic longitude (top panel) and latitude (bottom panel) for the nonclassified sources, $\mathrm{H}$ in regions, $\mathrm{PNe}$, and pulsars. For $\mathrm{H}$ in regions we plot only the sources associated with the THOR continuum sources. For PNe and pulsars, we plot all that lie within our survey area.

spectral index maps for sources larger than that should be interpreted with caution. Figure 12 shows the spectral index maps of three jet sources. As the figure shows, the spectral index varies within the source, indicating that the optical depth of the synchrotron emission varies or possible thermal emission exists in different parts of the radio lobes.

In Sect. 5.2 we mentioned that the spatial distribution and spectral index distribution of the unresolved sources indicate the majority are extragalactic sources. To further confirm this, we compare the normalized spatial distribution and spectral index distribution of the non-classified sources to the Galactic sources we identified. Figure 13 compares the normalized distribution along Galactic longitude and latitude of those nonclassified sources with the Galactic objects ( $\mathrm{H}$ II regions, $\mathrm{PNe}$, and pulsars). The normalized distribution along Galactic longitude (top-panel, Fig. 13) shows that the Galactic objects are more concentrated in the lower longitude region, while more non-classified sources are detected in the higher longitude region. The distribution along Galactic latitude (top panel, Fig. 13) shows that the $\mathrm{H}$ II regions are concentrated very close to the Galactic midplane. Although not as much as the $\mathrm{H}$ II regions, the $\mathrm{PNe}$ and pulsars are also concentrated close to the midplane. In contrast, more nonclassified sources are found in regions $|b|>0.5^{\circ}$. This distribution

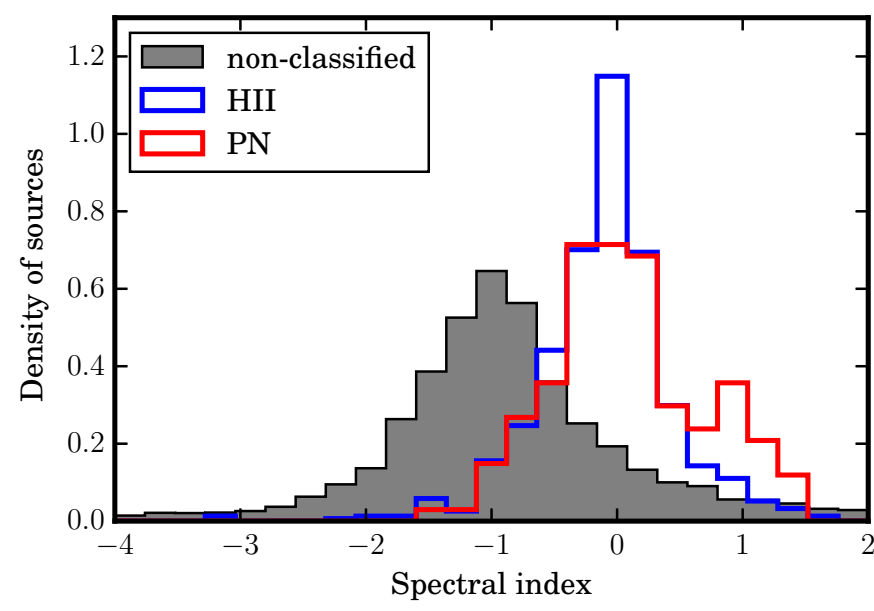

Fig. 14. Normalized spectral index distribution of the non-classified sources, $\mathrm{H}_{\text {II }}$ regions, and $\mathrm{PNe}$.

difference confirms that the non-classified sources are of mostly extragalactic origin. Furthermore, the spectral index distribution of the non-classified sources also shows clear differences from the Galactic sources and peaks around $\alpha \sim-1$ (Fig. 14), but many of these non-classified sources still show a flat SED $(\alpha \gtrsim 0)$, which could be Galactic compact $\mathrm{H}$ II regions that are not listed in the WISE H II catalog. We classify the $\sim 5300$ sources with a negative spectral index $\alpha<-0.3$ as extragalactic source candidates, among which $\sim 3970$ sources have a reliable spectral index (fit_spws $\geq 4$ ).

Extragalactic radio sources often consist of a core and one or more radio lobes, which show different spectral profiles. The core dominates the emission at shorter wavelengths and shows a close to flat or positive spectral index, while the jet dominates at longer wavelengths and shows negative spectral index (e.g., Hey 1971, chap. 9). This transition in the spectral profile happens around $\sim 10-20 \mathrm{~cm}$. Assuming the emission of the core and jet at $1-2 \mathrm{GHz}$ can both be described with one single power law, then the total flux $I(v)$ is proportional to $\left(c_{1} v^{\alpha_{1}}+c_{2} v^{\alpha_{2}}\right)$, where $\alpha_{1}$ and $\alpha_{2}$ are the spectral indices of the jet and core, respectively, and $c_{1}$ and $c_{2}$ are constants. We used the Markov chain Monte Carlo method (MCMC) to fit the extragalactic source candidates that have a $\mathrm{S} / \mathrm{N}$ larger than 50 with the two spectral indices using the emcee package (Foreman-Mackey et al. 2013). Then we selected the candidates that meet the following criteria for a good fit: first, the reduced $\chi^{2}$ of the fitting of two spectral indices is smaller than that with single spectral index fitting; second, the spectra of the two components cross with each other within our frequency coverage (1.05-1.95 GHz, such as Fig. 15). In total, we can derive a good fit with two spectral indices for 135 continuum sources.

We performed an $F$-test (e.g., Phillips 1982; Lomax \& Hahs-Vaughn 2013) to compare the two components fitting to the one power-law fitting, and calculated the probability values ( $p$-value) for all 135 sources. The $p$-values are all above 0.28 , therefore the two components fitting is not significantly better than the simple one power-law fitting. However, the two components fitting results still provide alternative insight for those sources. As shown in Fig. 15, the peak fluxes of G50.695-0.792 can be fitted with two components with a spectral indices of -1.47 and 0.03 , respectively, which could trace the radio jet and core, respectively. The averaged image in Fig. 15 shows G50.695-0.792 is resolved with an elongated structure, which might trace the jet. 

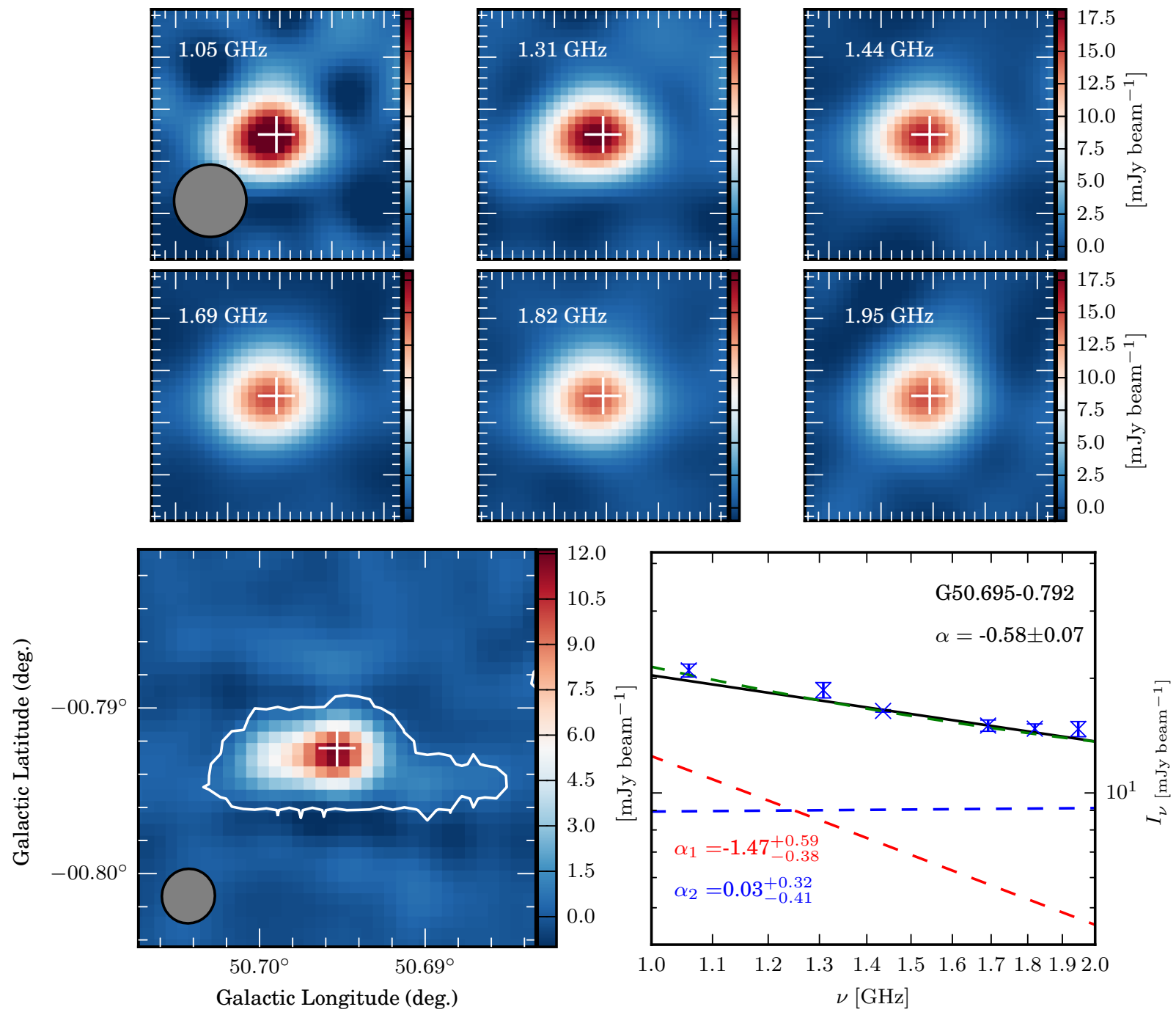

Fig. 15. One example source G50.695-0.792 fitted with two indices. The top two panels show each SPW separately. The bottom left panel represents the averaged image of spw-1440 and spw-1820, which we used for the source extraction (see Sect. 3). The white contours show the extent of the source determined by the BLOBCAT algorithm. The cross in each panel marks the peak position, which we used to determine the spectral index. The bottom right panel presents the peak intensity for each SPW and corresponding one single spectral index fitting (black solid line) and two spectral indices fitting (green dashed lines) from two components (red and blue dashed lines).

Table 5. Summary of different types of sources in the catalog.

\begin{tabular}{lc}
\hline \hline Source types & Number of continuum sources \\
\hline H II regions & 713 \\
SNRs & $92(+21)$ \\
PNe & 164 \\
Pulsars & $38(+663)$ \\
X-ray sources & 79 \\
Extragalactic jets & 299 \\
USS & 699 \\
\hline
\end{tabular}

Notes. USS stands for sources with ultra steep spectra $(\alpha \leq-1.3)$. The numbers of the candidates are in brackets.

Radio sources with ultra steep spectra (USS; $\alpha \leq-1.3$ ) are efficient tracers of high-redshift radio galaxies, since radio sources can be detected uniformly over all redshift ranges and do not suffer dust extinction at high redshifts (e.g., Chambers et al. 1996; Hughes et al. 1997; Ivison et al. 1998; De Breuck et al.
2000). De Breuck et al. (2000) found a trend that steeper spectral index sources have higher redshifts, and $50 \%$ of the 4 C USS sample (Chambers et al. 1996) are $z>2$ sources. This $z-\alpha$ correlation is considered to be a combined contribution of a K-correction and an increasing spectral curvature with $z$ of a radio spectrum (Krolik \& Chen 1991; Carilli et al. 1999; van Breugel et al. 1999).

In our continuum catalog, we have $\sim 2170$ sources that have an spectral index steeper than -1.3 , which is our base sample of USS sources. De Breuck et al. (2000) further pointed out that $85 \%$ USS with X-ray detections are in galaxy clusters, so we chose those without X-ray emission. Furthermore, we selected sources with a reliable spectral index determination (fit_spws $\geq 4$ ). This selection results in 1362 sources that have a good spectral index determination and have a spectral index steeper than -1.3 . Of these source 663 have a spectral index steeper than -1.6 and could be Galactic pulsars (De Breuck et al. 2000). The remaining 699 USS sources could be high-redshift radio galaxies; further spectroscopic observations are needed to confirm this. 


\section{Conclusions}

We observed a large portion of the first Galactic quadrant $(l=$ $\left.14.0-67.4^{\circ},|b| \leq 1.25^{\circ}\right)$ using the VLA in C-configuration and achieved a spatial resolution of $\sim 10-25^{\prime \prime}$ at 1 to $2 \mathrm{GHz}$ with the THOR Galactic plane survey. In this paper, we present the catalog of the continuum sources from the whole survey. We summarise numbers of different types of sources in the catalog in Table 5 and the main results below.

1. The catalog contains 10387 sources we extracted with the BLOBCAT software after removing the obvious observational side-lobe artifacts. About $72 \%$ (7521 sources) of the extracted sources are detected at a significance higher than $7 \sigma$ and $\sim 79 \%$ are unresolved. The catalog is complete to at least $94 \%$ above the $7 \sigma$ detection limit. The noise of our data is dominated by side-lobe noise and varies spatially, although more than $60 \%$ of the observed area has a noise level of $7 \sigma \lesssim 2 \mathrm{mJy} \mathrm{beam}^{-1}$. We extracted the peak intensity of the 6 usable SPWs between 1 to $2 \mathrm{GHz}$, and we are able to determine a reliable spectral index (spectral index fitted with at least 4 SPWs) for 5657 sources.

2. We cross-matched the THOR catalog with the WISE H II region catalog and found 713 continuum sources are associated with $\mathrm{H}$ in regions. Among the matched $\mathrm{H}$ in regions, 16 are in the radio quiet group in the WISE catalog, which means they did not previously have radio continuum detected. We find that 231 continuum sources are associated with more than one $\mathrm{H}_{\text {II }}$ region. The spectral index distribution shows a single peak around $\alpha=0$, indicating thermal free-free emission. For 168 sources we fit the SED with a simple homogeneous $\mathrm{H}$ II region model and derive the EM and the electron density $\left(n_{\mathrm{e}}\right)$ where the distance information is available in the WISE catalog.

3. Although the diffuse emission from many of the large scale SNRs is filtered out by our interferometric observations, we identified 92 continuum sources associated with 39 SNRs from the SNR catalog by Green (2014). Thirteen of the new SNR candidates from Anderson et al. (2017) are detected in our continuum catalog.

4. By cross-matching the THOR catalog with the HASH database, we detected $164 \mathrm{PNe}$ in our continuum catalog. As 90 of these $\mathrm{PNe}$ do not have radio emission information at $20 \mathrm{~cm}$ in the database, our survey provides this important information in Table D.1. The spectral index distribution is similar to that of the $\mathrm{H}_{\text {II }}$ regions and shows a single peak around $\alpha=0$, indicating thermal free-free emission.

5. We cross-matched the THOR catalog with the ATNF Pulsar Catalog and found 38 counterparts. One extremely intermittent pulsar J1841-0500 is also detected in our catalog. We find that 663 sources with a spectral index $\alpha<-1.6$ could be Galactic pulsar candidates.

6. We cross-matched the THOR catalog with X-ray source catalogs 1SXPS, 3XMM- DR7 and CSC and found 79 overlaps. Twelve of these have a spectral index steeper than -1.3 and could be galaxy clusters. Forty-three do not have previous known radio counterparts within a radius of $15^{\prime \prime}$ on SIMBAD or NED.

7. About 300 sources show clear structure of bipolar jets; we denote these as jets in the catalog and construct spectral index maps. We identified 699 USS sources and these could be highredshifted radio galaxies $(-1.3>\alpha>-1.6)$. Further spectroscopic observations are needed to confirm this.

8. About 9000 sources in our catalog are not classified specifically. They are likely to be extragalactic background sources.
More than 7750 sources do not have counterparts in the SIM-

BAD Astronomical Database and more than 3760 sources do not have counterparts in the NED.

With the THOR continuum catalog, we provide a rich dataset to the community. All the fits images and catalogs can be downloaded from the project website ${ }^{14}$. With the follow-up observations of particular sources, such as absorption study of the extragalactic sources, or by combining with other existing Galactic plane surveys ${ }^{15}$, we can study the the structure of the Milky Way and the ISM in different phases.

Acknowledgements. The National Radio Astronomy Observatory is a facility of the National Science Foundation operated under cooperative agreement by Associated Universities, Inc. Y. W., H. B., S. B., and J. D. S. acknowledge support from the European Research Council under the Horizon 2020 Framework Program via the ERC Consolidator Grant CSF-648505, and RSK via the ERC AdvancedGrant 339177 (STARLIGHT). H.B. and M.R. acknowledge support from the Deutsche Forschungsgemeinschaft in the Collaborative Research Center (SFB 881) "The Milky Way 74 System" (subproject B1, B2, B8), and from the Priority Program SPP 1573 "Physics of the Interstellar Medium" (grant numbers KL 1358/18.1, KL 1358/19.2). F.B. acknowledges funding from the European Union's Horizon 2020 research and innovation programme (grant agreement No 726384). The authors thank J. Mottram, M. Fouesneau and K. Meisenheimer for productive and fruitful discussions. This research made use of Astropy and affiliated packages, a community-developed core Python package for Astronomy (Astropy Collaboration 2018), Python package SciPy, APLpy, an open-source plotting package for Python (Robitaille \& Bressert 2012), and software TOPCAT (Taylor 2005).

\section{References}

Aguirre, J. E., Ginsburg, A. G., Dunham, M. K., et al. 2011, ApJS, 192, 4 Anderson, L. D., Bania, T. M., Balser, D. S., et al. 2014, ApJS, 212, 1 Anderson, L. D., Wang, Y., Bihr, S., et al. 2017, A\&A, 605, A58 Astropy Collaboration, Price-Whelan, A. M., \& Sipőcz, B. M. 2018, AJ, 156, 123

Balser, D. S., Rood, R. T., Bania, T. M., \& Anderson, L. D. 2011, ApJ, 738, 27 Benjamin, R. A., Churchwell, E., Babler, B. L., et al. 2003, PASP, 115, 953 Beuther, H., Bihr, S., Rugel, M., et al. 2016, A\&A, 595, A32

Bhatnagar, S., Rau, U., Green, D. A., \& Rupen, M. P. 2011, ApJ, 739, L20

Bihr, S., Beuther, H., Ott, J., et al. 2015, A\&A, 580, A112

Bihr, S., Johnston, K. G., Beuther, H., et al. 2016, A\&A, 588, A97

Bojičić, I. S., Parker, Q. A., Filipović, M. D., \& Frew, D. J. 2011, MNRAS, 412, 223

Bridle, A. H., \& Perley, R. A. 1984, ARA\&A, 22, 319

Brisken, W. F., Romani, R. W., \& Ng, C. Y. 2005, in X-Ray and Radio Connections, eds. L. O. Sjouwerman, \& K. K. Dyer, 5.04

Camilo, F., Ransom, S. M., Chatterjee, S., Johnston, S., \& Demorest, P. 2012, ApJ, 746, 63

Carey, S. J., Noriega-Crespo, A., Mizuno, D. R., et al. 2009, PASP, 121, 76

Carilli, C. L., Röttgering, H. J. A., Miley, G. K., Pentericci, L. H., \& Harris, D. E. 1999, in The Most Distant Radio Galaxies, eds. H. J. A. Röttgering, P. N. Best, \& M. D. Lehnert

Chambers, K. C., Miley, G. K., van Breugel, W. J. M., \& Huang, J.-S. 1996, ApJS, 106, 215

Churchwell, E., Babler, B. L., Meade, M. R., et al. 2009, PASP, 121, 213

Condon, J. J. 1992, ARA\&A, 30, 575

Condon, J. J., Cotton, W. D., Greisen, E. W., et al. 1998, AJ, 115, 1693

Csengeri, T., Urquhart, J. S., Schuller, F., et al. 2014, A\&A, 565, A75

De Breuck, C., van Breugel, W., Röttgering, H. J. A., \& Miley, G. 2000, A\&AS, 143,303

Decourchelle, A. 2005, in X-Ray and Radio Connections, eds. L. O. Sjouwerman, \& K. K. Dyer, 4.02

Evans, I. N., Primini, F. A., Glotfelty, K. J., et al. 2010, ApJS, 189, 37

Evans, P. A., Osborne, J. P., Beardmore, A. P., et al. 2014, ApJS, 210, 8

Foreman-Mackey, D., Hogg, D. W., Lang, D., \& Goodman, J. 2013, PASP, 125, 306

Green, D. A. 2014, Bull. Astron. Soc. India, 42, 47

Grobler, T. L., Nunhokee, C. D., Smirnov, O. M., van Zyl, A. J., \& de Bruyn, A. G. 2014, MNRAS, 439, 4030

Hales, C. A., Murphy, T., Curran, J. R., et al. 2012, MNRAS, 425, 979

\footnotetext{
14 http://www . mpia.de/thor/Overview.html
}

15 https://www. scipy.org/ 
Harris, D. E., \& Krawczynski, H. 2006, ARA\&A, 44, 463

Helfand, D. J., Becker, R. H., White, R. L., Fallon, A., \& Tuttle, S. 2006, AJ, 131,2525

Hey, J. S. 1971, The radio universe

Hoare, M. G., Purcell, C. R., Churchwell, E. B., et al. 2012, PASP, 124, 939

Hughes, D. H., Dunlop, J. S., \& Rawlings, S. 1997, MNRAS, 289, 766

Ivison, R. J., Dunlop, J. S., Hughes, D. H., et al. 1998, ApJ, 494, 211

Kalcheva, I. E., Hoare, M. G., Urquhart, J. S., et al. 2018, A\&A, 615, A103

Keto, E. 2003, ApJ, 599, 1196

Krolik, J. H., \& Chen, W. 1991, AJ, 102, 1659

Lomax, R., \& Hahs-Vaughn, D. 2013, Statistical Concepts : A Second Course (Taylor \& Francis)

Lucas, P. W., Hoare, M. G., Longmore, A., et al. 2008, MNRAS, 391, 136

Luo, S. G., Condon, J. J., \& Yin, Q. F. 2005, ApJS, 159, 282

Maccarone, T. J., Torres, M. A. P., Britt, C. T., et al. 2012, MNRAS, 426 3057

Manchester, R. N., Hobbs, G. B., Teoh, A., \& Hobbs, M. 2005, AJ, 129, 1993

Miller, N. A., Neff, S. G., \& Mushotzky, R. F. 2005, in X-Ray and Radio

Connections, eds. L. O. Sjouwerman, \& K. K. Dyer, 5.05

Mirabel, I. F., \& Rodríguez, L. F. 1998, Nature, 392, 673

Mirabel, I. F., \& Rodríguez, L. F. 1999, ARA\&A, 37, 409

Parker, Q. A., Bojičić, I. S., \& Frew, D. J. 2016, J. Phys. Conf. Ser., 728, 032008

Phillips, P. C. B. 1982, Biometrika, 69, 261

Purcell, C. R., Hoare, M. G., Cotton, W. D., et al. 2013, ApJS, 205, 1

Reynoso, E. M., \& Walsh, A. J. 2015, MNRAS, 451, 3044
Robitaille, T., \& Bressert, E. 2012, Astrophysics Source Code Library [record ascl:1208.017]

Rosen, S. R., Webb, N. A., Watson, M. G., et al. 2016, A\&A, 590, A1 Rosolowsky, E., Dunham, M. K., Ginsburg, A., et al. 2010, ApJS, 188, 123

Rugel, M. R., Beuther, H., Bihr, S., et al. 2018, A\&A, 618, A159

Rybicki, G. B., \& Lightman, A. P. 1979, Radiative processes in astrophysics (New York: Wiley-Interscience), 393

Sánchez-Monge, Á 2011, PhD Thesis

Schuller, F., Menten, K. M., Contreras, Y., et al. 2009, A\&A, 504, 415

Seaquist, E. R. 1993, Rep. Prog. Phys., 56, 1145

Silich, S. 2005, in X-Ray and Radio Connections, eds. L. O. Sjouwerman, \& K. K. Dyer, 3.03

Stil, J. M., Taylor, A. R., Dickey, J. M., et al. 2006, AJ, 132, 1158

Taylor, M. B. 2005, in Astronomical Data Analysis Software and Systems XIV, eds. P. Shopbell, M. Britton, \& R. Ebert, Astronomical Society of the Pacific Conference Series, 347, 29

Tetarenko, B. E., Bahramian, A., Arnason, R. M., et al. 2016, ApJ, 825, 10

Urquhart, J. S., Thompson, M. A., Moore, T. J. T., et al. 2013, MNRAS, 435, 400

van Breugel, W., Breuck, C., \& Stanford, S. A. 1999, The Hy-Redshift Universe: Galaxy Formation and Evolution at High Redshift. Astronomical Society of the Pacific Conference Series., 193, 44

Watson, M. G., Schröder, A. C., Fyfe, D., et al. 2009, A\&A, 493, 339

Wilson, T. L., Rohlfs, K., \& Hüttemeister, S. 2013, Tools of Radio Astronomy (Heidelberg: Springer-Verlag Berlin) 
Y. Wang et al.: Radio continuum emission in the northern Galactic plane: Sources and spectral indices from the THOR survey

\section{Appendix A: Noise maps}
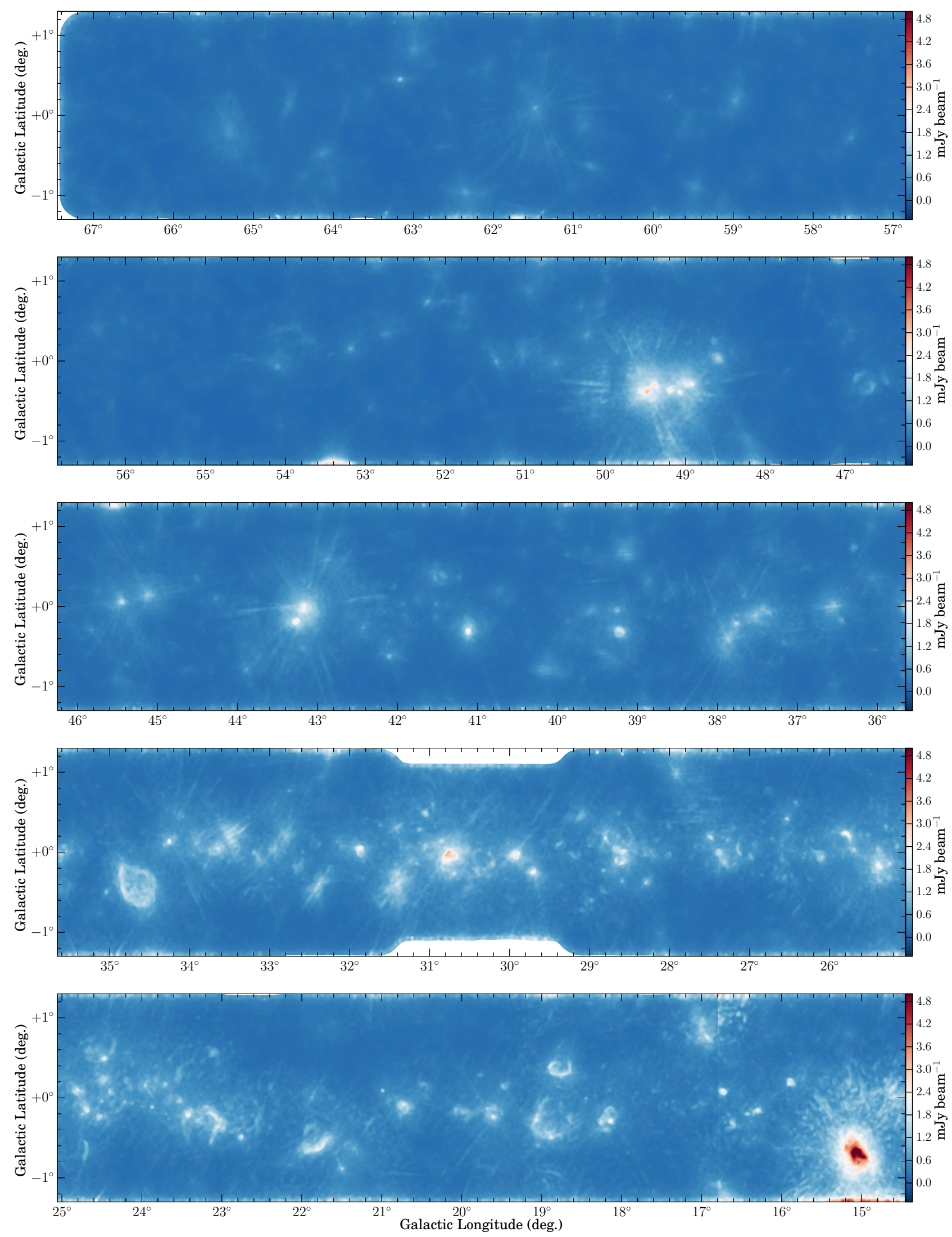

Fig. A.1. Noise map of the THOR survey using the average of spw-1440 and spw-1820. 


\section{Appendix B: Completeness maps}
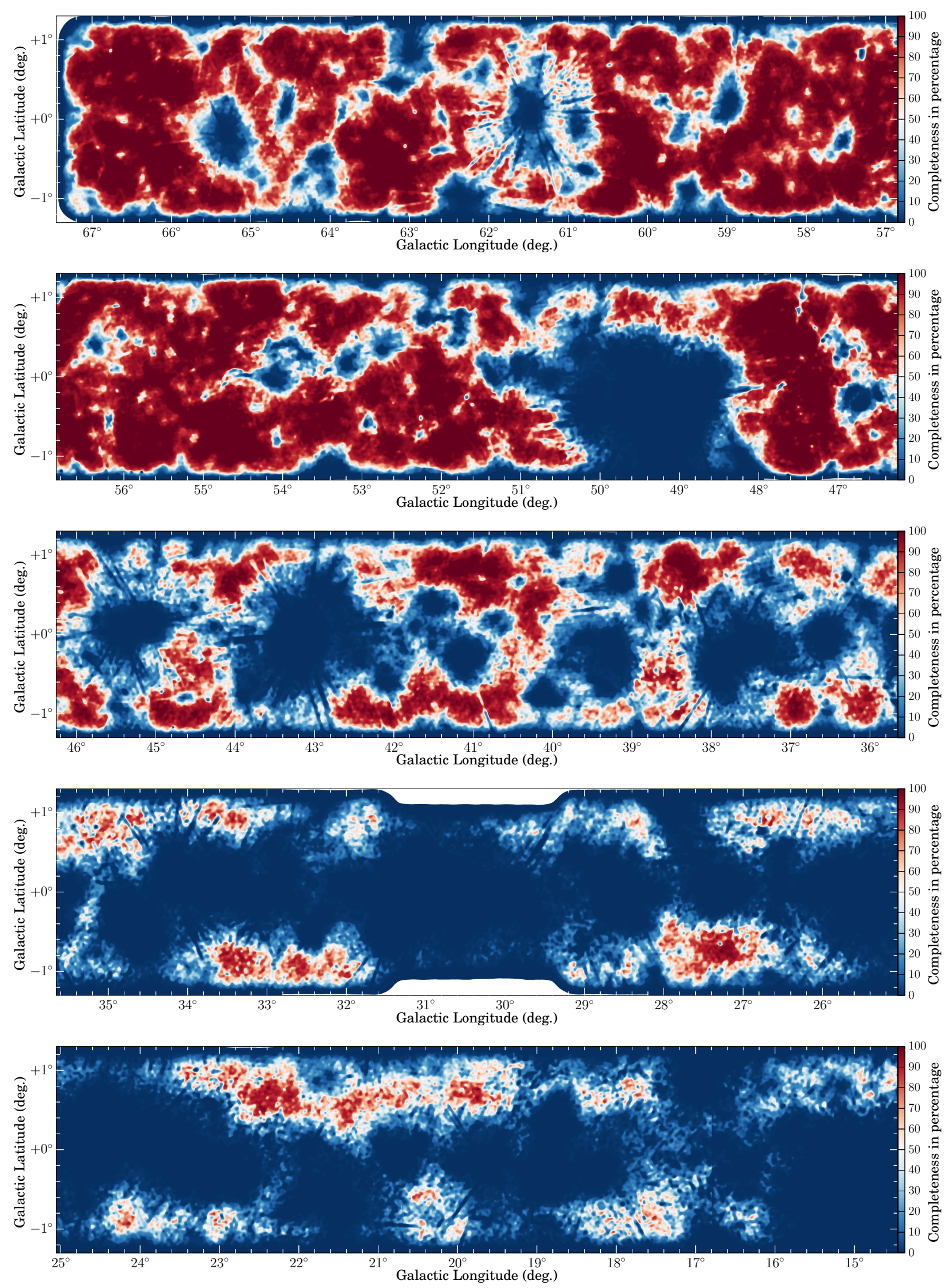

Fig. B.1. Completeness map in percentage for sources with a peak intensity of $1 \mathrm{mJy} \mathrm{beam}^{-1}$. 
Y. Wang et al.: Radio continuum emission in the northern Galactic plane: Sources and spectral indices from the THOR survey
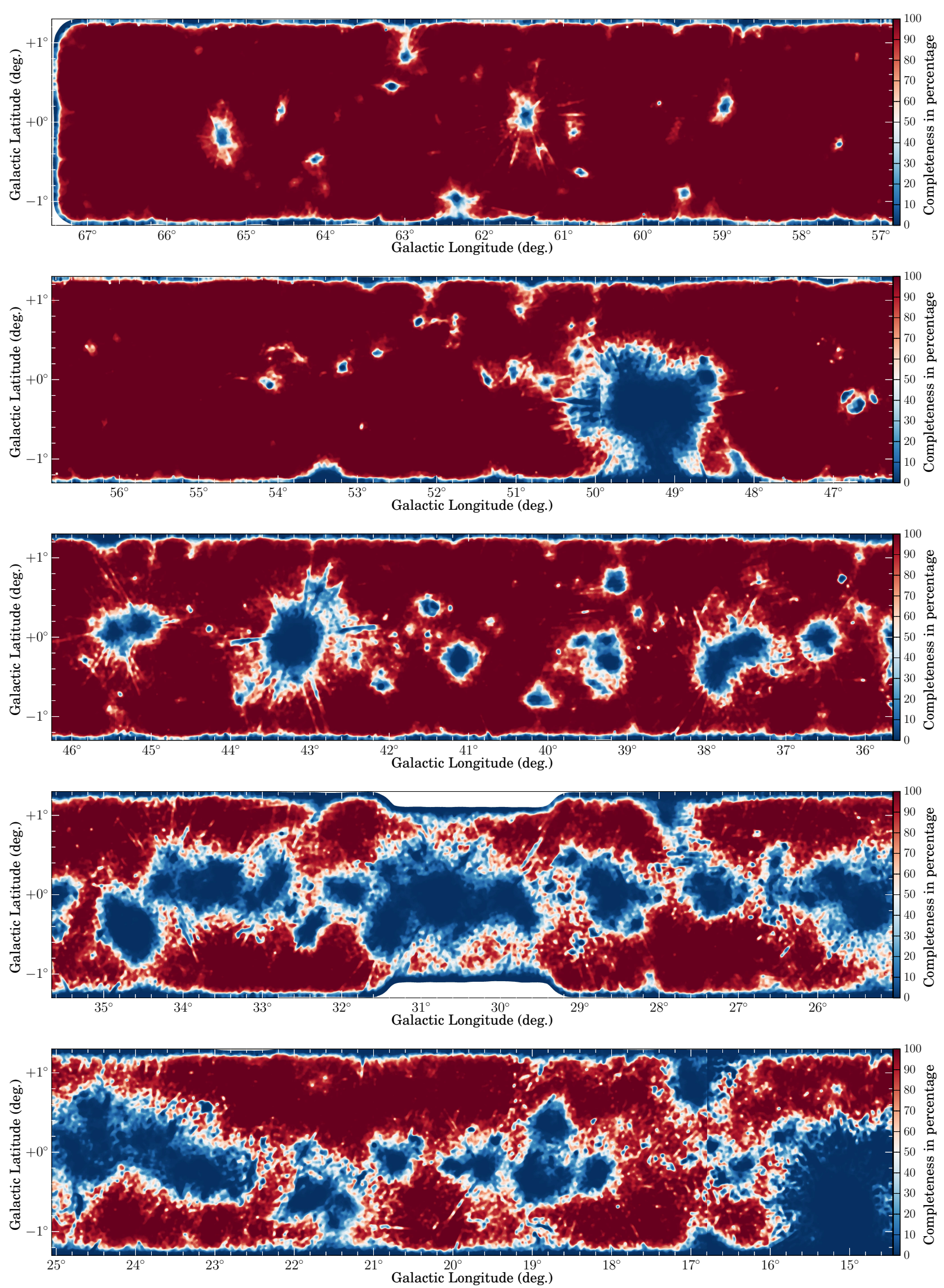

Fig. B.2. Completeness map in percentage for sources with a peak intensity of $2 \mathrm{mJy}_{\text {beam }}{ }^{-1}$. 

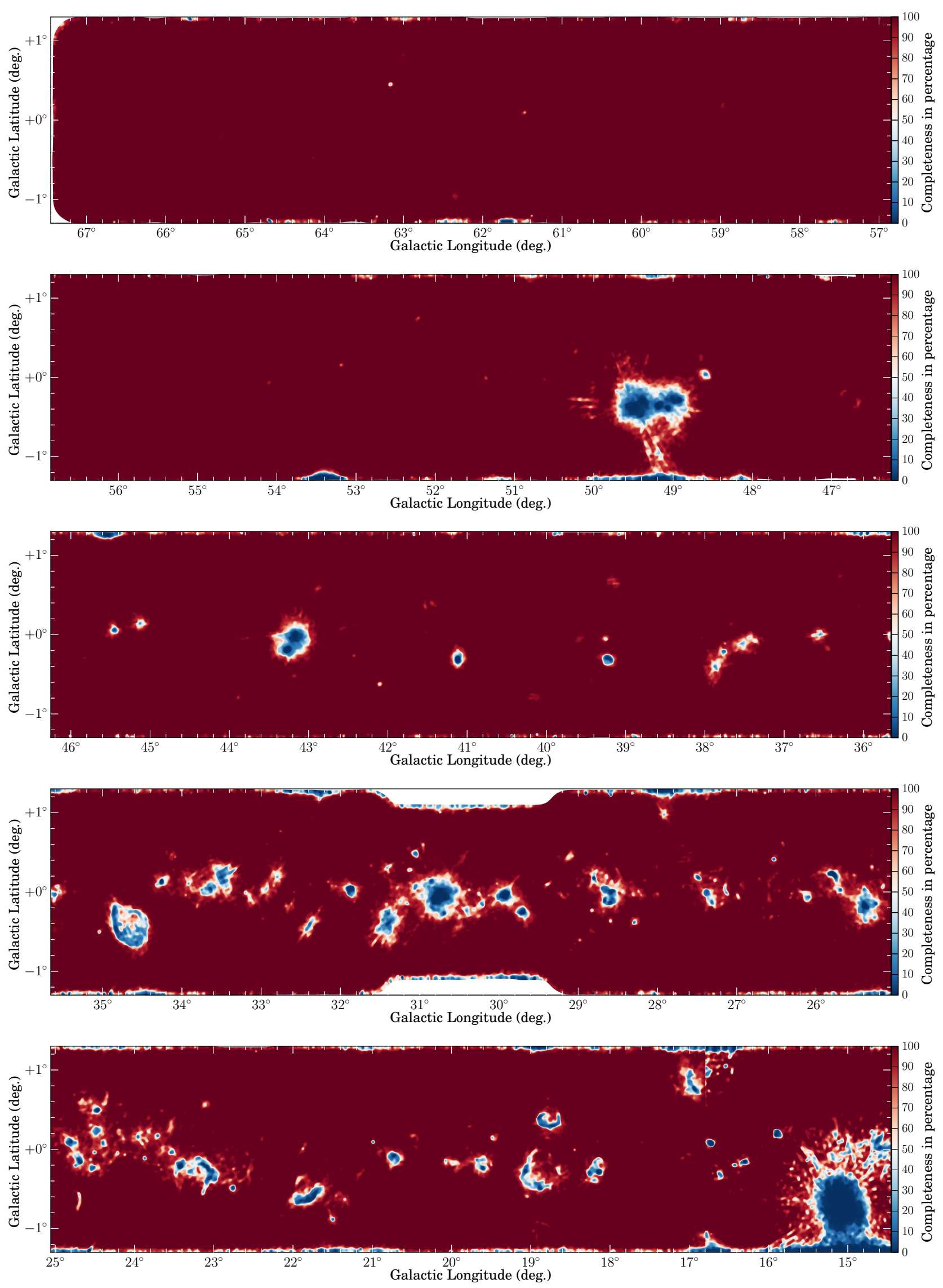

Fig. B.3. Completeness map in percentage for sources with a peak intensity of $5 \mathrm{mJy} \mathrm{beam}^{-1}$. 
Y. Wang et al.: Radio continuum emission in the northern Galactic plane: Sources and spectral indices from the THOR survey
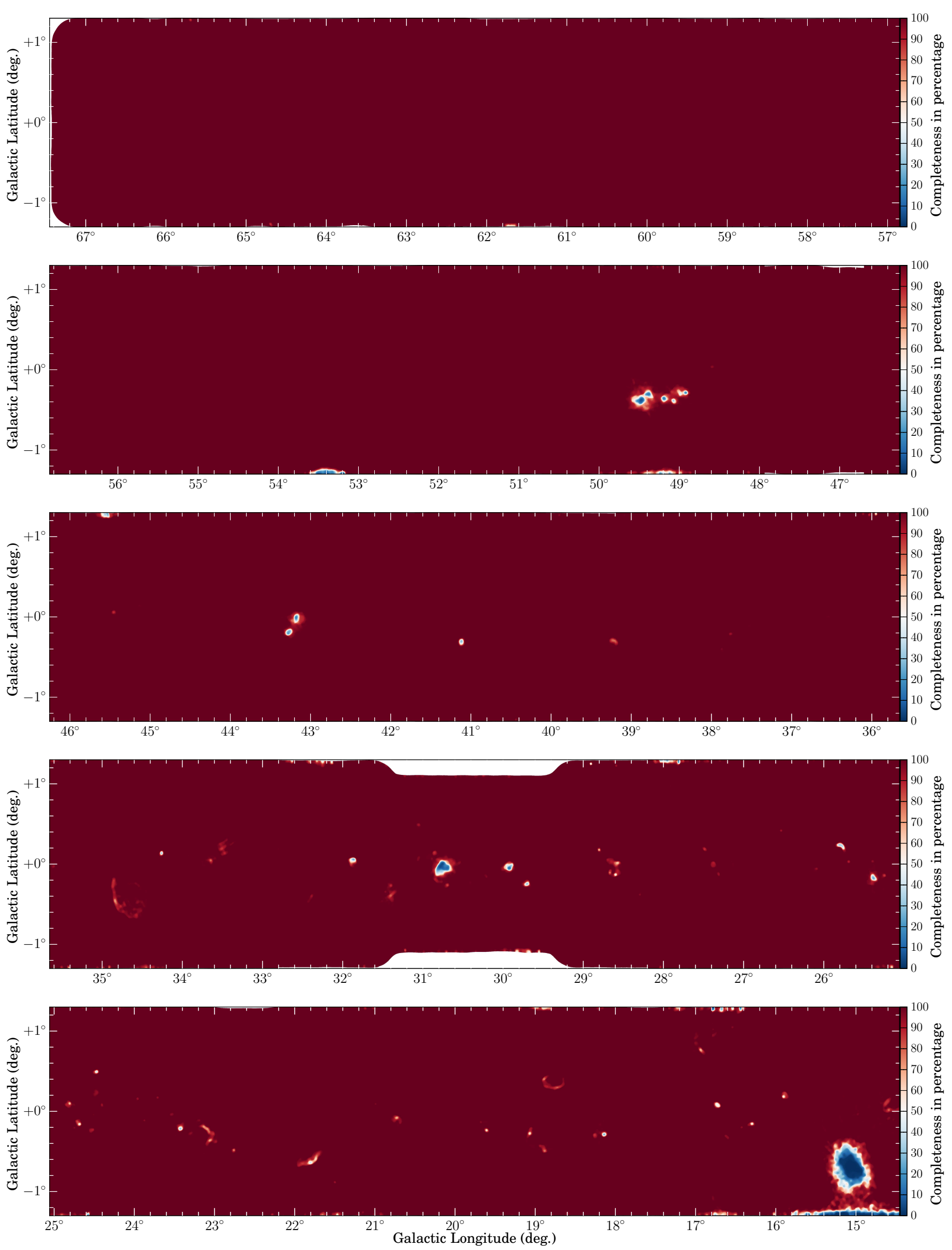

Fig. B.4. Completeness map in percentage for sources with a peak intensity of $10 \mathrm{mJy} \mathrm{beam}^{-1}$. 


\section{Appendix C: Compare with CORNISH}

Sources with a positive spectral index between 1 to $2 \mathrm{GHz}$ are mostly dominated by optically thick free-free emission at these frequencies. The emission, however, should be optically thin at higher frequencies and should flatten the spectral index there (e.g., Sánchez-Monge 2011). If we estimate the flux density at $5 \mathrm{GHz}$ for these sources, we could easily overestimate the flux. Since we do not have flux density measurements between $2 \mathrm{GHz}$ and $5 \mathrm{GHz}$, we are not sure whether such overestimation would be due to the uncertainty of spectral index or optical depth variation.

Sources with a spectral index $\lesssim-0.5$ are mostly dominated by synchrotron emission. Depending on the nature of the source, the spectral index could get flatter between $2 \mathrm{GHz}$ and $5 \mathrm{GHz}$ as a possible contribution from free-free emission could increase (e.g., Condon 1992) since free-free emission has a flat or positive spectral index. If we estimate the flux density at $5 \mathrm{GHz}$ for these sources, we would underestimate the flux.

Sources with a flat spectral index $(\sim-0.1)$ in the THOR frequency range are dominated by optically thin free-free emission. The emission at $5 \mathrm{GHz}$ would still be dominated by optically thin free-free emission, since the dust continuum is not relevant until $\sim 100 \mathrm{GHz}$ and synchrotron emission contribution is even smaller than at the lower frequencies. Thus, we can estimate the flux density at $5 \mathrm{GHz}$ from the THOR flux density measurements properly and reliably.

Among the 1905 THOR sources that are matched with a CORNISH source within a radius of $5^{\prime \prime}, 1087$ sources are unresolved and are detected in all six SPWs in THOR (fit_spws =6). We extrapolated flux densities at $5 \mathrm{GHz}$ for all these 1087 sources according to their spectral indices from the THOR catalog and plotted against the flux densities in the CORNISH catalog; see Fig. C.1. Figure C.1 shows that comparing to the flux densities in the CORNISH catalog, as expected we overestimate the flux density for many sources with a positive spectral index, and

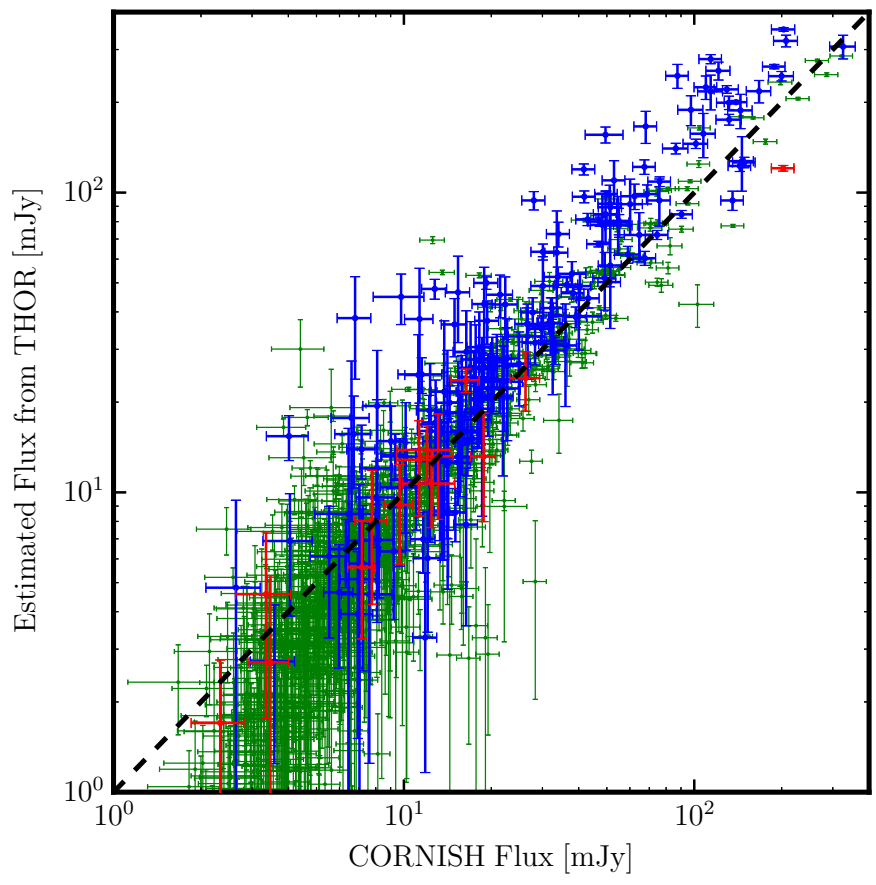

Fig. C.1. Extrapolated flux densities at $5 \mathrm{GHz}$ for all 1087 compact sources that are detected in all 6 SPWs in THOR (fit_spws =6) according to their spectral indices from the THOR catalog plotted against the flux densities in the CORNISH catalog. The green crosses indicate sources with a spectral index smaller than 0 , the blue crosses represent those with a spectral index larger than 0 , and the red crosses indicate sources with a spectral index between -0.09 and -0.11 (optically thin free-free emission). The dashed line represents a one-to-one relation.

similarly we underestimate the flux density for many sources with a negative spectral index. This is expected for such a general extrapolation for the reasons explained above. 


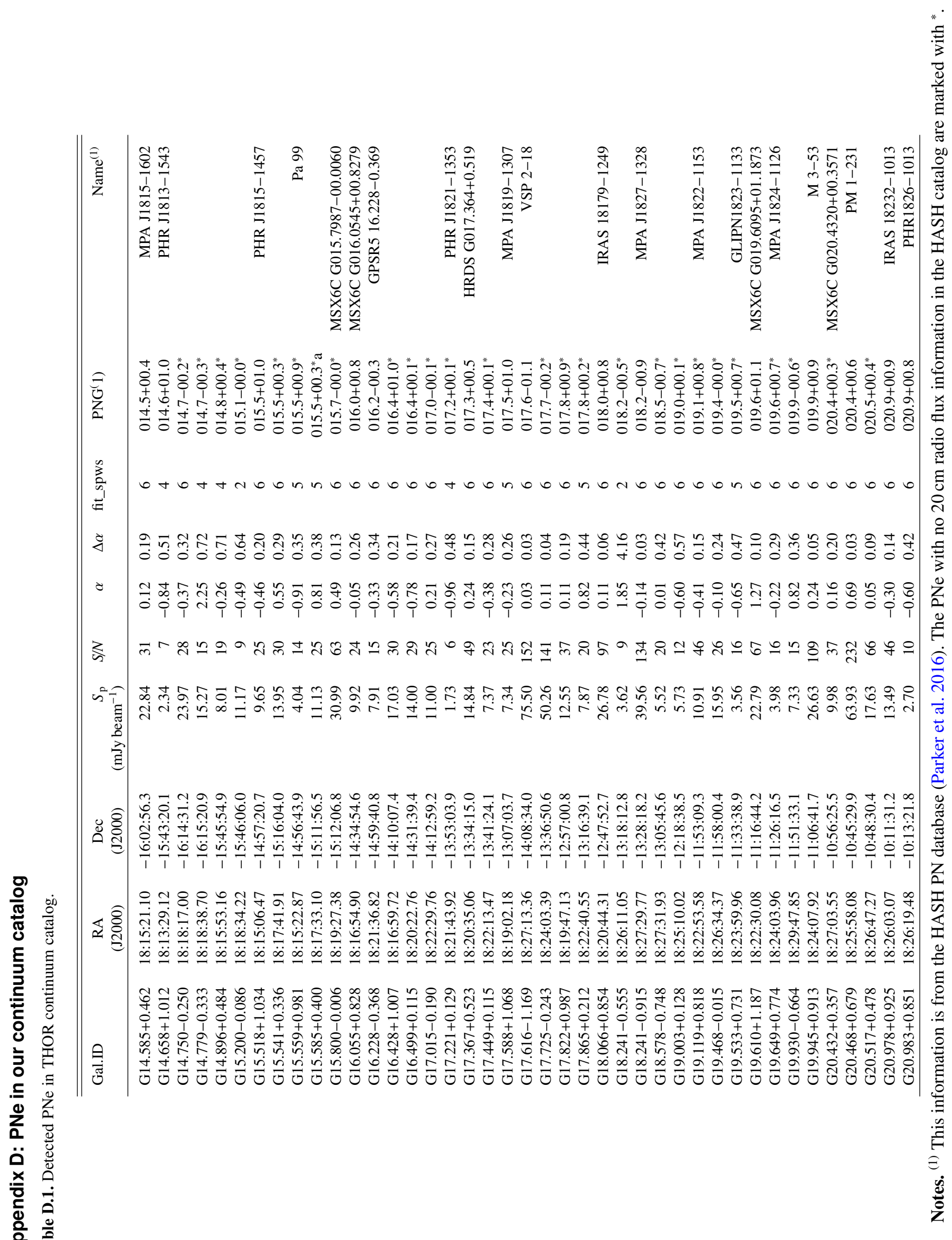




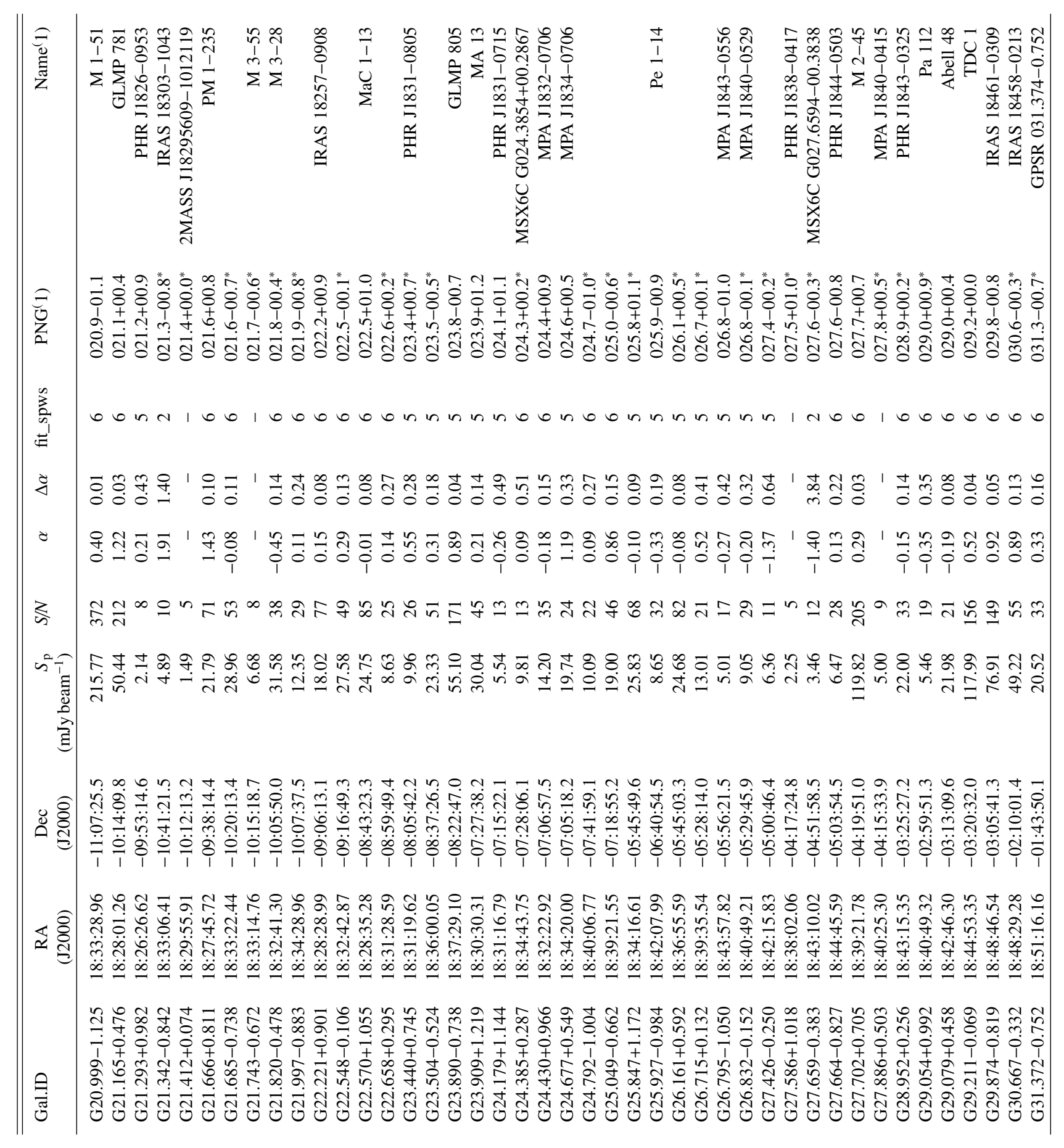




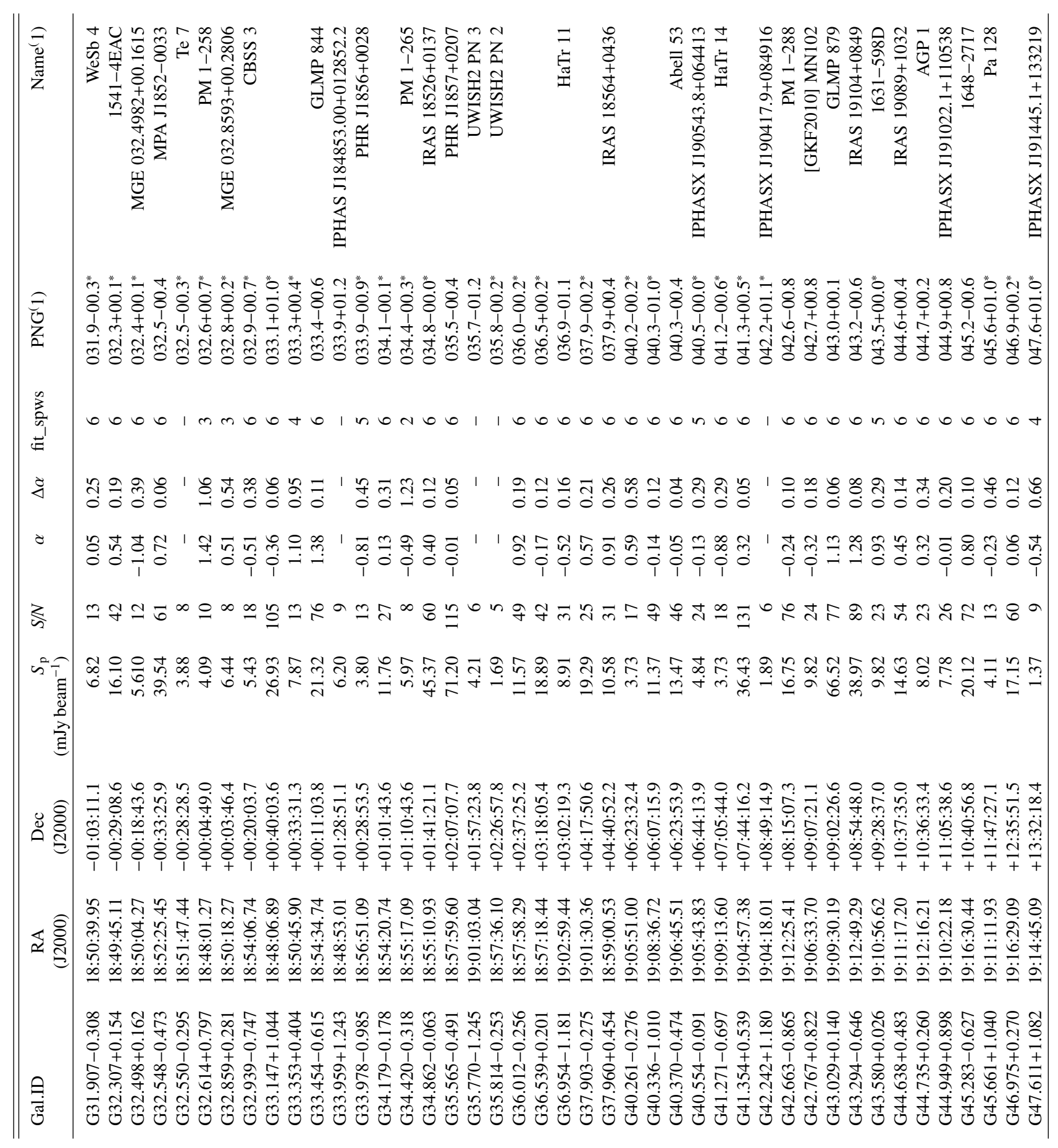




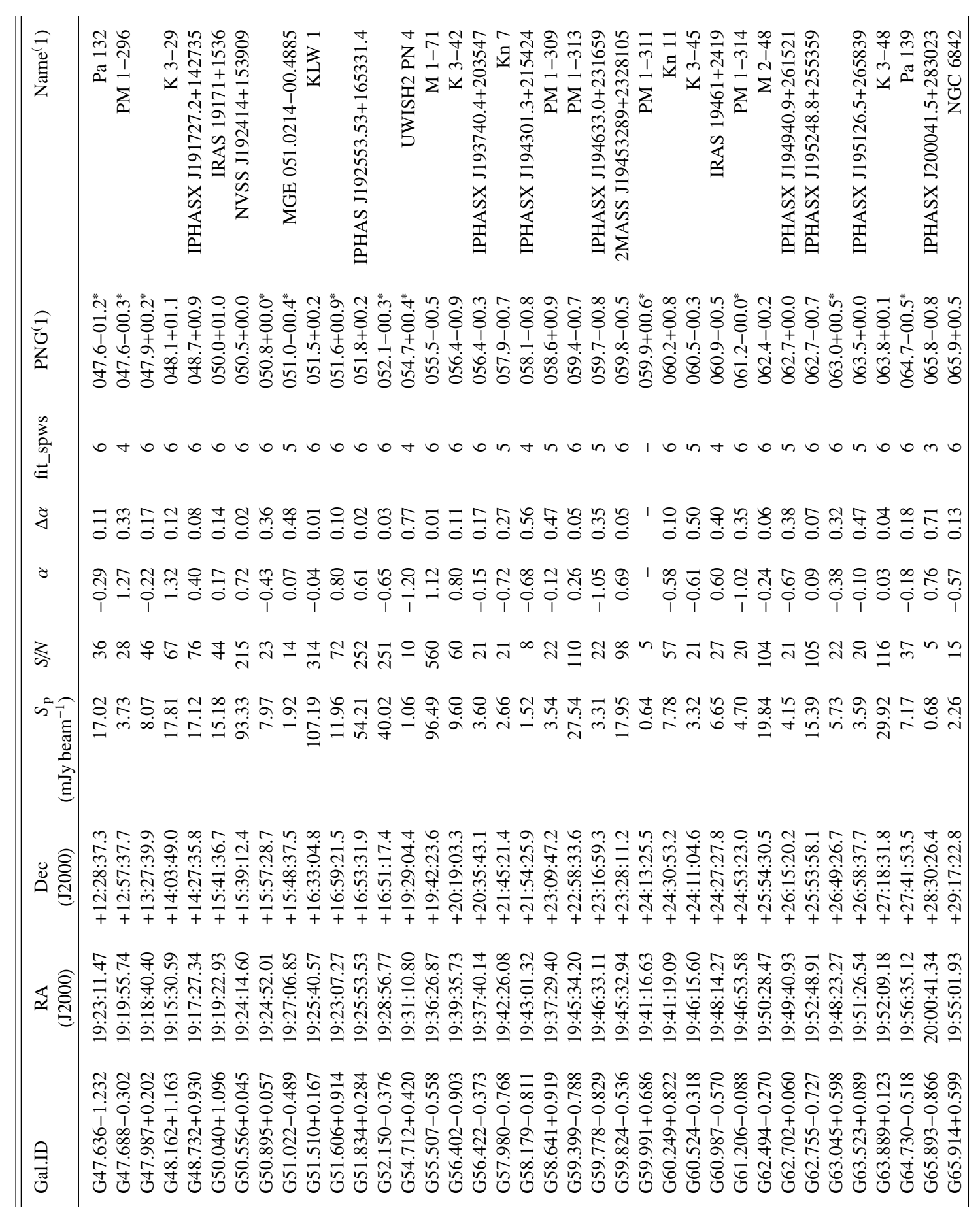


Y. Wang et al.: Radio continuum emission in the northern Galactic plane: Sources and spectral indices from the THOR survey

\section{Appendix E: Detected pulsars in our continuum catalog}

Table E.1. Detected pulsars in our continuum catalog

\begin{tabular}{|c|c|c|c|c|c|c|c|c|}
\hline Gal.ID & $\begin{array}{c}\text { RA } \\
(\mathrm{J} 2000)\end{array}$ & $\begin{array}{c}\text { Dec } \\
(\mathrm{J} 2000)\end{array}$ & $\begin{array}{r}S_{\mathrm{p}} \\
\left(\mathrm{mJy}_{\text {beam }}{ }^{-1}\right)\end{array}$ & $S / N$ & $\alpha$ & $\Delta \alpha$ & fit_spws & PSR Name \\
\hline $\mathrm{G} 16.405+0.610$ & $18: 18: 23.69$ & $-14: 22: 38.1$ & 5.60 & 14 & -2.95 & 0.30 & 6 & B1815-14 \\
\hline $\mathrm{G} 17.160+0.483$ & $18: 20: 19.69$ & $-13: 46: 17.0$ & 2.40 & 7 & - & - & - & B1817-13 \\
\hline G18.001-0.691 & $18: 26: 13.18$ & $-13: 34: 47.9$ & 3.55 & 16 & -1.29 & 0.48 & 5 & B1823-13 \\
\hline G19.767+0.946 & $18: 23: 40.30$ & $-11: 15: 10.5$ & 3.48 & 17 & -1.55 & 0.32 & 6 & B1820-11 \\
\hline G19.810+0.741 & $18: 24: 29.54$ & $-11: 18: 40.0$ & 1.01 & 6 & -1.05 & 1.73 & 2 & B $1821-11$ \\
\hline $\mathrm{G} 21.286+0.798$ & $18: 27: 05.49$ & $-09: 58: 43.4$ & 1.74 & 8 & -2.72 & 4.08 & 2 & B $1824-10$ \\
\hline $\mathrm{G} 23.385+0.063$ & $18: 33: 40.15$ & $-08: 27: 32.1$ & 4.89 & 8 & -1.35 & 1.10 & 3 & B1830-08 \\
\hline $\mathrm{G} 25.172+0.762$ & $18: 34: 29.38$ & $-06: 33: 03.3$ & 1.77 & 5 & 0.06 & 1.36 & 2 & J1834-0633 \\
\hline G27.073-0.941 & $18: 44: 05.09$ & $-05: 38: 32.7$ & 1.93 & 9 & -2.03 & 0.59 & 4 & B 1841-05 \\
\hline G27.322-0.033 & $18: 41: 17.95$ & $-05: 00: 21.6$ & 6.27 & 6 & - & - & - & $\mathrm{J} 1841-0500$ \\
\hline $\mathrm{G} 27.818+0.279$ & $18: 41: 05.74$ & $-04: 25: 21.6$ & 2.11 & 6 & 0.97 & 1.57 & 2 & B1838-04 \\
\hline G28.193-0.785 & $18: 45: 34.76$ & $-04: 34: 29.2$ & 2.19 & 8 & 0.4 & 1.93 & 2 & B1842-04 \\
\hline $\mathrm{G} 28.347+0.174$ & $18: 42: 26.55$ & $-03: 59: 59.6$ & 6.83 & 12 & -1.88 & 0.89 & 3 & B1839-04 \\
\hline G28.876-0.939 & $18: 47: 22.78$ & $-04: 02: 15.3$ & 3.01 & 10 & -1.90 & 0.60 & 3 & B1844-04 \\
\hline $\mathrm{G} 31.339+0.039$ & $18: 48: 23.56$ & $-01: 23: 58.9$ & 10.64 & 14 & -1.15 & 0.77 & 3 & B1845-01 \\
\hline $\mathrm{G} 32.763+0.091$ & $18: 50: 48.27$ & $-00: 06: 31.1$ & 12.99 & 17 & -0.66 & 0.35 & 6 & J1850-0006 \\
\hline $\mathrm{G} 36.007+0.057$ & $18: 56: 50.89$ & $+02: 45: 45.4$ & 1.50 & 6 & -2.84 & 5.76 & 2 & $\mathrm{~J} 1856+0245$ \\
\hline G37.213-0.637 & $19: 01: 31.72$ & $+03: 31: 05.4$ & 3.27 & 9 & -3.21 & 1.17 & 2 & B $1859+03$ \\
\hline G38.163-0.151 & $19: 01: 32.37$ & $+04: 35: 08.1$ & 5.45 & 17 & -2.86 & 0.31 & 5 & $\mathrm{~J} 1901+0435$ \\
\hline G39.814+0.335 & $19: 02: 50.39$ & & 1.52 & 7 & -4.68 & 0.64 & 2 & B1900+06 \\
\hline $\mathrm{G} 40.569+1.056$ & $19: 01: 39.01$ & $+07: 16: 34.4$ & 1.78 & 10 & -1.00 & 0.82 & 4 & B $1859+07$ \\
\hline G40.604-0.304 & $19: 06: 35.18$ & $+06: 41: 03.9$ & 2.27 & 10 & - & - & - & B1904+06 \\
\hline G40.944+0.065 & $19: 05: 53.73$ & $+07: 09: 19.0$ & 1.25 & 6 & - & - & - & B1903+07 \\
\hline G41.520-0.871 & $19: 10: 18.84$ & $+07: 14: 09.5$ & 1.07 & 5 & -0.89 & 1.23 & 2 & $\mathrm{~J} 1910+0714$ \\
\hline G41.740-0.772 & $19: 10: 22.07$ & $+07: 28: 35.8$ & 1.19 & 8 & -1.08 & 1.31 & 2 & $\mathrm{~J} 1910+0728$ \\
\hline G43.501-0.684 & $19: 13: 20.74$ & $+09: 04: 42.2$ & 2.61 & 7 & - & - & - & J1913+0904 \\
\hline G44.557-1.019 & $19: 16: 32.43$ & $+09: 51: 26.8$ & 1.53 & 11 & 0.01 & 2.34 & 2 & B1914+09 \\
\hline G44.707-0.650 & $19: 15: 29.98$ & $+10: 09: 43.9$ & 1.15 & 7 & -2.09 & 3.31 & 2 & $\mathrm{~B} 1913+10$ \\
\hline G44.832+0.992 & $19: 09: 48.76$ & $+11: 02: 02.5$ & 1.75 & 9 & 0.51 & 1.12 & 3 & B $1907+10$ \\
\hline $\mathrm{G} 47.576+0.451$ & $19: 16: 58.69$ & $+13: 12: 49.4$ & 1.84 & 18 & -0.73 & 0.48 & 5 & B1914+13 \\
\hline $\mathrm{G} 48.260+0.624$ & $19: 17: 39.80$ & $+13: 53: 56.6$ & 4.68 & 33 & -1.74 & 0.22 & 6 & B $1915+13$ \\
\hline G49.096+0.866 & $19: 18: 23.54$ & $+14: 45: 04.0$ & 2.10 & 13 & -0.45 & 1.08 & 3 & B $1916+14$ \\
\hline G51.859+0.063 & $19: 26: 45.32$ & $+16: 48: 32.0$ & 1.42 & 10 & 0.76 & 1.37 & 3 & B $1924+16$ \\
\hline G55.575+0.639 & $19: 32: 07.99$ & $+20: 20: 47.6$ & 0.88 & 7 & -3.67 & 3.30 & 2 & B $1929+20$ \\
\hline G57.509-0.290 & $19: 39: 38.59$ & $+21: 34: 58.7$ & 11.05 & 21 & -3.78 & 0.13 & 6 & B $1937+21$ \\
\hline G57.903+0.308 & $19: 38: 14.12$ & $+22: 13: 11.6$ & 0.74 & 5 & 2.73 & 1.29 & 2 & $\mathrm{~J} 1938+2213$ \\
\hline G65.839+0.443 & $19: 55: 27.97$ & $+29: 08: 43.4$ & 1.13 & 10 & -0.69 & 1.62 & 3 & B $1953+29$ \\
\hline G65.924+0.772 & $19: 54: 22.47$ & $+29: 23: 16.3$ & 8.10 & 43 & -3.45 & 0.13 & 6 & B $1952+29$ \\
\hline
\end{tabular}




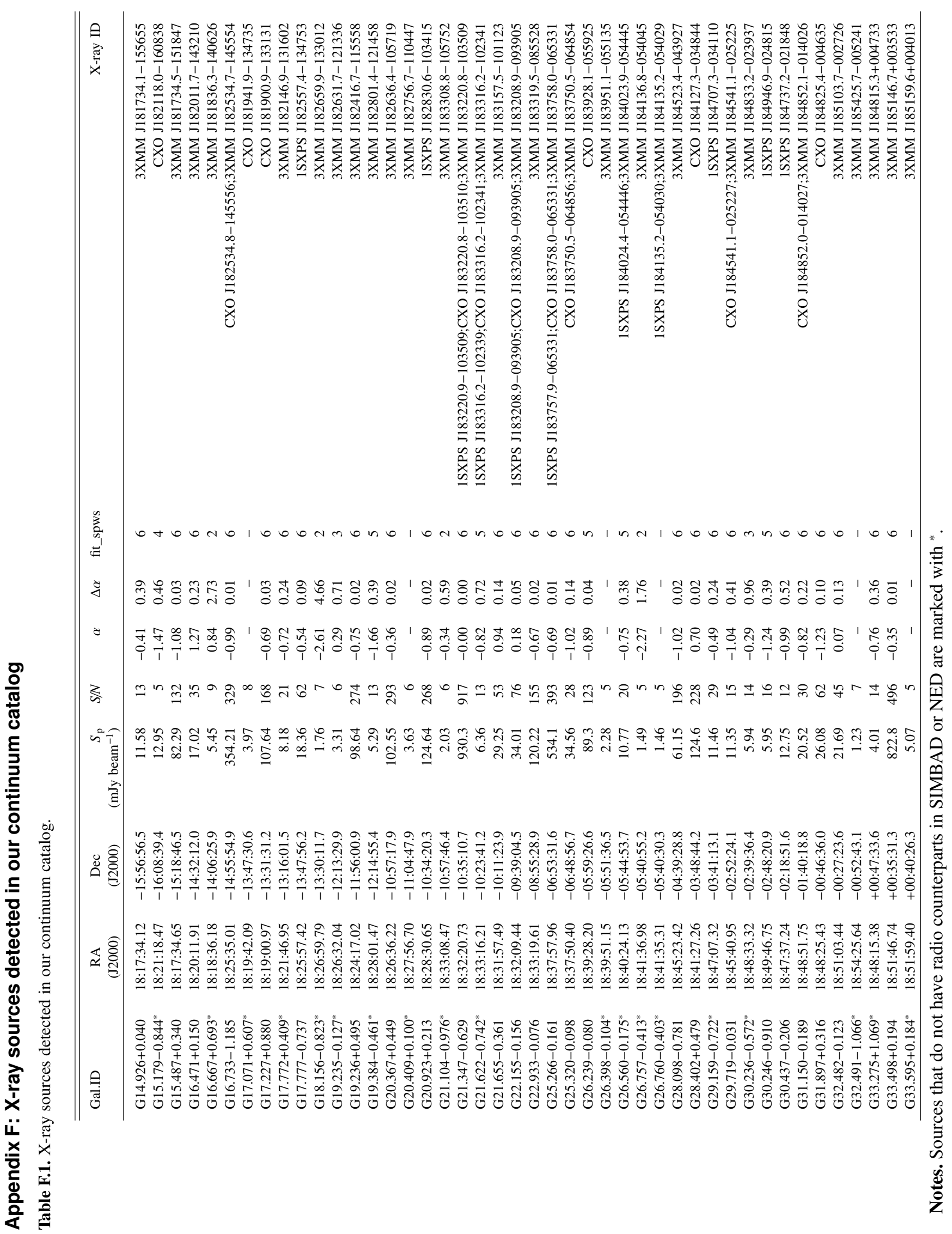




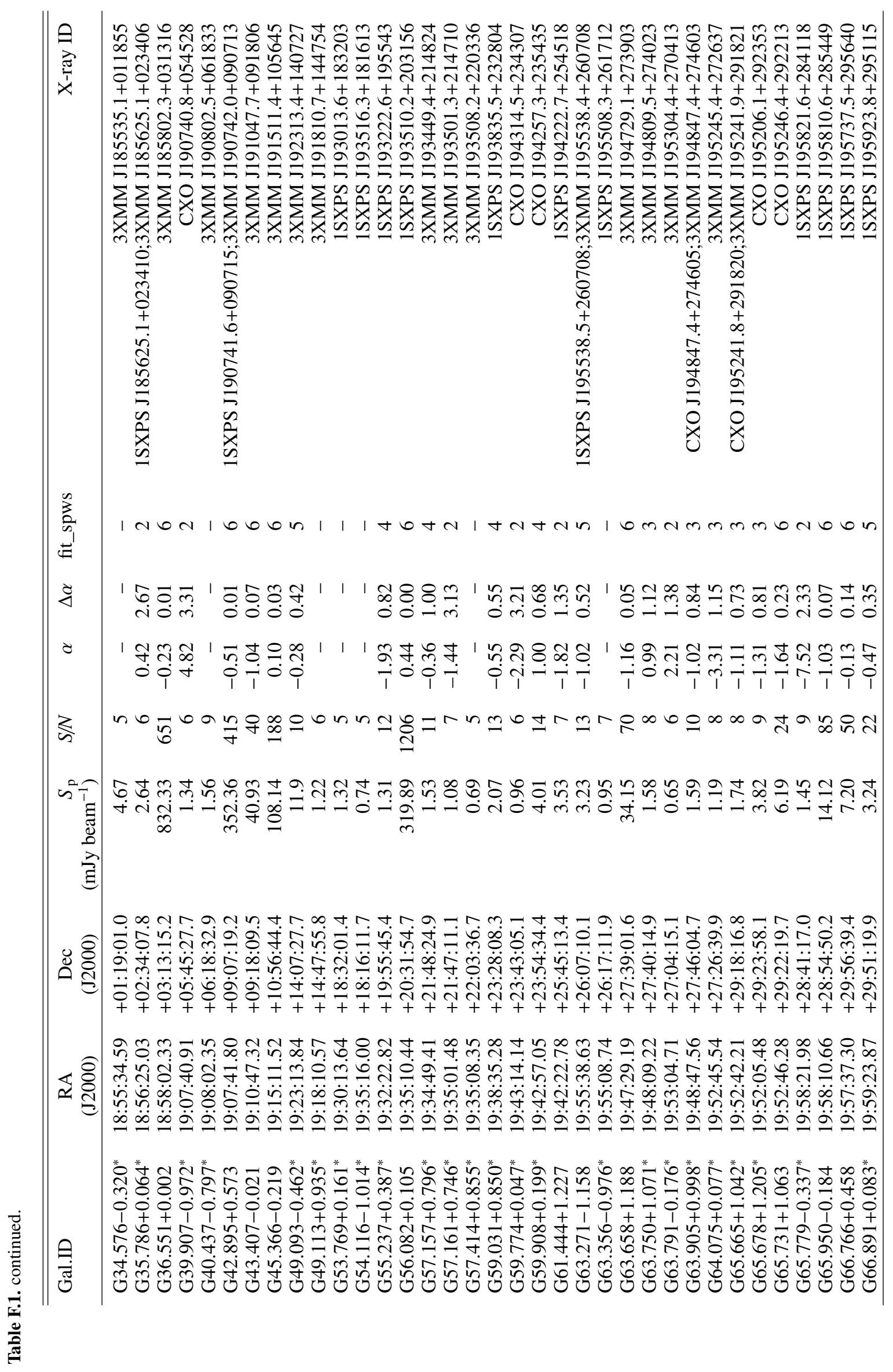

